\title{
UNIVERSAL MEASURE ZERO, LARGE HAUSDORFF DIMENSION, AND NEARLY LIPSCHITZ MAPS
}

\author{
ONDŘEJ ZINDULKA
}

\begin{abstract}
We prove that each analytic set in $\mathbb{R}^{n}$ contains a universally null set of the same Hausdorff dimension and that each metric space contains a universally null set of Hausdorff dimension no less than the topological dimension of the space. Similar results also hold for universally meager sets.

An essential part of the construction involves an analysis of Lipschitzlike mappings of separable metric spaces onto Cantor cubes and selfsimilar sets.
\end{abstract}

\section{INTRODUCTION}

A separable metric space $X$ is universally null (or has universal measure zero) if $\mu(X)=0$ for each finite Borel measure $\mu$ on $X$ that is diffused (i.e. vanishes on singletons). This is obviously equivalent to each Borel diffused measure $\mu$ on $X$ being degenerate, in that for each Borel set $B \subseteq X$ one has either $\mu(B)=0$ or $\mu(B)=\infty$. In particular, on a universally null space the $s$-dimensional Hausdorff measure is degenerate for all $s>0$.

Can such a set have positive Hausdorff or even topological dimension? Fremlin $[13,439 \mathrm{G}]$ has an example of a subset $E$ of the plane whose 1dimensional Hausdorff measure is infinite and degenerate on $E$. In [41] it is proved that universally null sets with positive topological dimension exist if, and only if, there is a universally null set $E \subseteq \mathbb{R}$ with the cardinality of continuum, and that there are universally null sets of arbitrary Hausdorff dimension.

The goal of the present paper is to get better results in this direction. Namely, to prove that some metric spaces, including all analytic sets in Euclidean spaces, contain a universally null subset with the same Hausdorff dimension.

We outline the basic ideas of our construction. Let $2^{\omega}$ be the usual Cantor space. Equip it with the metric given by $d(x, y)=2^{-n}$, where $n$

2000 Mathematics Subject Classification. 28A78, 54E35, 54F45.

Key words and phrases. Universally null, universally meager, Hausdorff dimension, upper Hausdorff dimension, Cantor cube, nearly Lipschitz mapping, monotone space.

This project was supported by Department of Education of the Czech Republic, research project BA MSM 210000010. 
is the first integer for which $x(n) \neq y(n)$, and with the standard product probability measure $\mu$. It turns out that $\mu$ is the one-dimensional Hausdorff measure on $2^{\omega}$ and thus the Hausdorff dimension of $2^{\omega}$ is 1 . By a famous result of Edwin Grzegorek there are two sets $A, B \subseteq 2^{\omega}$ that have the same cardinality but are of different sizes from the measure-theoretic viewpoint: $A$ has positive (outer) measure and $B$ is universally null. A diagonal set $D \subseteq A \times B \subseteq 2^{\omega} \times 2^{\omega}$ has positive one-dimensional Hausdorff measure: for the projection of $D$ onto $A$ is Lipschitz. It is also universally null: for the projection onto $B$ is one-to-one, and a continuous one-to-one preimage of a universally null set is universally null.

But how to get such a set in $2^{\omega}$ ? Can one copy this diagonal set into $2^{\omega}$ in such a way that Hausdorff dimension of the copy is still large? Yes indeed, there is a homeomorphism of $2^{\omega}$ onto $2^{\omega} \times 2^{\omega}$ that is not quite Lipschitz, yet "Lipschitz enough" to give the preimage of the diagonal set $D$ Hausdorff dimension 1 . The preimage of $D$ is universally null, because $D$ is. So $2^{\omega}$ contains a universally null set of full Hausdorff dimension. This method works for a class of generalized Cantor sets.

To get a universally null set of full Hausdorff dimension in $\mathbb{R}$ one can just approximate $\mathbb{R}$ from within by sufficiently thick Cantor sets. And it turns out that this approximation can be achieved in a wide class of metric spaces that are, in a sense, linearly ordered (so called monotone spaces, cf. Section 4), and include e.g. all analytic subsets of $\mathbb{R}$ and all analytic ultrametric spaces.

Employing classical projection and intersection theorems one can extend the construction to Euclidean spaces. And using [41, Lemma 5.1] on topological dimension and Lipschitz maps yields, in any metric space $X$, a universally null set of Hausdorff dimension no less than the topological dimension of $X$.

The method can be executed in a more abstract setting (Section 5), obtaining thus similar results for universally meager sets (Section 7) and some other classes of small sets (Section 8).

Cantor and self-similar sets, monotone spaces, and the ways Lipschitz and Lipschitz-like maps act between them (Sections 3 and 4) receive more attention than necessary for our purposes. It is because the notions seem to be interesting in their own right.

\section{Preliminaries}

The set of all natural numbers including zero is denoted $\omega$. The cardinality of the continuum is denoted $\mathfrak{c}$. The cardinality of a set $A$ is denoted $|A|$. The power set of $A$ is denoted $\mathcal{P}(A)$. 
Metric spaces. Most (though not all) metric spaces under consideration are separable. Recall that a metric space is perfect if it has no isolated points, Polish if it is separable and completely metrizable, and analytic if it is a continuous image of a Polish space.

In order to avoid unnecessary technicalities, all metric spaces are assumed to be bounded. This is no constraint, since any metric $d$ can be redefined to be bounded e.g. by $d_{1}=\min (d, 1)$, which leaves close distances unaffected.

Within a context of a metric space we use some self-explanatory notation, e.g. diam $E$ denotes the diameter of a set $E$ and $\operatorname{dist}(A, B)$ the lower distance from a set $A$ to a set $B$.

A metric $d$ on a metrizable space $X$ is said to be compatible if it induces the topology of $X$.

Hausdorff measure. Recall that given $s \geqslant 0$, the $s$-dimensional Hausdorff measure $\mathcal{H}^{s}$ on a metric space $X$ is defined thus: For each $\delta>0$ and $E \subseteq X$ set

$$
\mathcal{H}_{\delta}^{s}(E)=\inf \sum_{n}\left(\operatorname{diam} E_{n}\right)^{s},
$$

where the infimum is taken over all finite or countable covers $\left\{E_{n}\right\}$ of $E$ by sets of diameter at most $\delta$, and put

$$
\mathcal{H}^{s}(E)=\sup _{\delta>0} \mathcal{H}_{\delta}^{s}(E) .
$$

The basic properties of $\mathcal{H}^{s}$ are well-known. It is an outer measure and its restriction to Borel sets is a $G_{\delta}$-regular Borel measure in $X$. General references: [11, 24, 31]. We shall need the following theorem of Howroyd [16] that generalizes earlier results of Besicovitch [4] and Davies [6]. We shall call it Davies Theorem.

Theorem 2.1 (Davies Theorem). Let $X$ be an analytic metric space and $s>0$. If $\mathcal{H}^{s}(X)>0$, then there is a compact set $K \subseteq X$ such that $0<\mathcal{H}^{s}(K)<\infty$.

We shall also need the famous Frostman lemma:

Theorem 2.2 (Frostman Lemma $[24,8.19]$ ). Let $X$ be an analytic metric space and $s>0$. If $\mathcal{H}^{s}(X)>0$, then there is a nontrivial finite Borel measure $\mu \leqslant \mathcal{H}^{s}$ on $X$ such that $\mu B(x, r) \leqslant r^{s}$ for each $x \in X, r>0$.

Hausdorff dimension. The Hausdorff dimension of $E$ is denoted and defined by

$$
\operatorname{dim}_{\mathbf{H}} E=\sup \left\{s: \mathcal{H}^{s}(E)>0\right\} .
$$

Hausdorff dimension is an intrinsic property. It is monotone, and countably stable in that $\operatorname{dim}_{\mathrm{H}} \bigcup_{n} E_{n}=\sup _{n} \operatorname{dim}_{\mathrm{H}} E_{n}$ for any countable family 
$\left\{E_{n}\right\}$ of sets. Hausdorff measures and dimension are preserved by Lipschitz preimages.

Lemma 2.3 ([11, Lemma 6.1]). If $f: X \rightarrow Y$ is Lipschitz and $\mathcal{H}^{s}(f X)>0$, then $\mathcal{H}^{s}(X)>0$, for each $s>0$. In particular, $\operatorname{dim}_{\boldsymbol{H}} X \geqslant \operatorname{dim}_{\mathrm{H}} f(X)$.

Upper Hausdorff dimension. We shall consider also the following variation of Hausdorff dimension. For a separable metric space $E$ let $E^{*}$ denote its completion and define the upper Hausdorff dimension of $E$ by

$$
\overline{\operatorname{dim}}_{\mathrm{H}} E=\inf \left\{\operatorname{dim}_{\mathrm{H}} K: E \subseteq K \subseteq E^{*}, K \text { is } \sigma \text {-compact }\right\} .
$$

Hence $\overline{\operatorname{dim}}_{H}$ is an intrinsic property. It is easy to see that for $E$ a subset of a complete metric space $X$ one may alternatively define

$$
\overline{\operatorname{dim}}_{\mathrm{H}} E=\inf \left\{\operatorname{dim}_{\mathrm{H}} K: E \subseteq K \subseteq X, K \text { is } \sigma \text {-compact }\right\},
$$

which shows that $\overline{\operatorname{dim}}_{H}$ is countably stable. See [39] for more on upper Hausdorff dimension including an equivalent definition based on Hausdorfflike measures.

Obviously $\overline{\operatorname{dim}}_{\mathrm{H}} \geqslant \operatorname{dim}_{\mathrm{H}}$ and if $E$ is $\sigma$-compact, then $\overline{\operatorname{dim}}_{\mathrm{H}} E=\operatorname{dim}_{\mathrm{H}} E$. On the other hand, there is a dense $G_{\delta^{-}}$set $E \subseteq \mathbb{R}$ such that $\operatorname{dim}_{H} E=0$, and any such set has by the following Lemma $2.4 \overline{\operatorname{dim}}_{H} E=1$. This show that $\operatorname{dim}_{H} E=\overline{\operatorname{dim}}_{H} E$ may easily fail. Note also that $\overline{\operatorname{dim}}_{H}$ is estimated from above by, but is in general not equal to, the lower packing dimension, and a fortiori by packing dimension.

The following simple lemma links upper Hausdorff dimension to category.

Lemma 2.4. Let $X$ be a separable metric space such that $\overline{\operatorname{dim}}_{\mathrm{H}} U \geqslant s$ for each nonempty open set $U$. If $E \subseteq X$ is nonmeager, then $\overline{\operatorname{dim}}_{\mathrm{H}} E \geqslant s$.

Proof. Notice that if $K$ is compact and $\operatorname{dim}_{H} K<s$, then $K$ is nowhere dense: For otherwise it would contain a nonempty open set $U$ and thus by assumption $\operatorname{dim}_{\mathrm{H}} K \geqslant \overline{\operatorname{dim}}_{\mathrm{H}} U \geqslant s$.

If $\overline{\operatorname{dim}}_{\mathrm{H}} E<s$, then there is a countable cover $\left\{K_{n}\right\}$ of $E$ by compact sets such that $\operatorname{dim}_{\mathrm{H}} K_{n}<s$ for each $n$. By the above, the sets $K_{n}$ are nowhere dense, whence $E$ is meager.

Nearly Lipschitz mappings. We shall make use of the following natural weakening of the notion of Lipschitz mapping.

Definition 2.5. A mapping $f:(X, d) \rightarrow(Y, \rho)$ between metric spaces is termed nearly Lipschitz if the following condition holds: For any $\varepsilon>0$ there is $\delta>0$ such that

$$
\rho(f(x), f(y)) \leqslant d(x, y)^{1-\varepsilon} \text { whenever } d(x, y)<\delta,
$$


i.e. if it is $\beta$-Hölder for each $\beta<1$. Equivalently, $f$ is nearly Lipschitz if there is a function $F:[0, \infty) \rightarrow[0, \infty)$ such that

$$
\rho(f(x), f(y)) \leqslant F(d(x, y)), \quad x, y \in X
$$

satisfying $\lim \sup _{r \rightarrow 0} r^{-\beta} F(r)=0$ for each $\beta<1$.

Every Lipschitz mapping is obviously nearly Lipschitz, but not vice versa. The growth condition for a nearly Lipschitz mapping is nevertheless good enough to control both Hausdorff dimensions the same way as Lipschitz mappings:

Lemma 2.6. If $f: X \rightarrow Y$ is nearly Lipschitz, then $\operatorname{dim}_{\mathrm{H}} X \geqslant \operatorname{dim}_{\mathrm{H}} f(X)$ and $\overline{\operatorname{dim}}_{\mathrm{H}} X \geqslant \overline{\operatorname{dim}}_{\mathrm{H}} f(X)$.

We shall use this easy lemma on several occasions. The first estimate follows easily e.g. from [31, Theorem 29], the second is similar.

\section{Cantor Cubes And SElF-Similar Sets}

Given a set $I$, denote by $I^{\omega}$ the set of all $I$-ary sequences and by $I^{<\omega}=$ $\bigcup_{n \in \omega} I^{n}$ the set off all finite $I$-ary sequences. Notice that for $p, q \in I^{<\omega}$, $p \subseteq q$ means that $q$ is an extension of $p$. If $p \in I \cup I^{<\omega}$ and $q \in I \cup I^{<\omega} \cup I^{\omega}$, the symbol $p * q$ denotes the concatenation. If $f \neq g$ are in $I^{\omega}$, the symbol $f \wedge g$ denotes the common initial segment of $f$ and $g$, and $n(f, g)=|f \wedge g|$ the length of $f \wedge g$. In more detail,

$$
\begin{aligned}
n(f, g) & =\min \{n \in \omega: f(n) \neq g(n)\}, \\
f \wedge g & =f\lceil n(f, g)=g\lceil n(f, g) .
\end{aligned}
$$

$(f \uparrow n$ is the restriction $=$ truncation of $f$.)

Cantor cubes. Fix $k=\{0,1, \ldots, k-1\} \in \omega$. Consider the cube $k^{\omega}$.

Let $\boldsymbol{r}=\left\langle r_{0}, r_{1}, \ldots, r_{k-1}\right\rangle \in(0,1)^{k}$. For each $p \in k^{<\omega}$ put

$$
\chi_{\boldsymbol{r}}(p)=\prod_{i<|p|} r_{p(i)} .
$$

The following formula defines a non-archimedean metric on $k^{\omega}$

$$
\rho_{\boldsymbol{r}}(f, g)= \begin{cases}\chi_{\boldsymbol{r}}(f \wedge g) & \text { if } f \neq g \\ 0 & \text { if } f=g\end{cases}
$$

that gives $k^{\omega}$ the standard product topology. The resulting metric space $\left(k^{\omega}, \rho_{\boldsymbol{r}}\right)$ is denoted by $\mathbb{C}(\boldsymbol{r})$. We often drop the subscript writing $\rho$ and $\chi$ for $\rho_{\boldsymbol{r}}$ and $\chi_{\boldsymbol{r}}$. 
It is easy to show (and follows at once from the theory of self-similar sets, see below), that the Hausdorff dimension of $\mathbb{C}(\boldsymbol{r})$ is the solution $s$ of Moran's equation

$$
\sum_{i<k} r_{i}^{s}=1
$$

and that $\mathcal{H}^{s}(\mathbb{C}(\boldsymbol{r}))=1$.

Definition 3.1. A metric space $\mathbb{C}$ is called a Cantor cube if there is $k \in \omega$ and $\boldsymbol{r} \in(0,1)^{k}$ such that $\mathbb{C}=\mathbb{C}(\boldsymbol{r})$.

Note that each Cantor cube is homeomorphic to $2^{\omega}$ ([21, Theorem 7.4]). The best known examples of a Cantor cube are $\mathbb{C}\left(\frac{1}{3}, \frac{1}{3}\right)$, which is bi-Lipschitz equivalent to the Cantor ternary set, and $\mathbb{C}\left(\frac{1}{2}, \frac{1}{2}\right)$. The latter has Hausdorff dimension one and its one-dimensional Hausdorff measure coincides on Borel sets with the standard product (Haar) measure.

Self-similar sets. Cantor cubes are particular instances of self-similar sets. We recall basic notions, the reader is referred to [34] for more details. Let $(X, d)$ be a complete metric space. A mapping $F: X \rightarrow X$ is called a similarity if there is a constant $r>0$ such that $d(F x, F y)=r \cdot d(x, y)$ for all $x, y \in X$. The constant $r$ is called the similarity ratio of $F$.

Let $\mathcal{F}=\left\langle F_{0}, F_{1}, \ldots, F_{k-1}\right\rangle$ be a finite sequence of similarities whose respective similarity ratios $\boldsymbol{r}=\left\langle r_{0}, r_{1}, \ldots, r_{k-1}\right\rangle$ are strictly less than 1 . Such a sequence is termed an Iterated Similarity System (ISS). The ISS $\mathcal{F}$ induces a set mapping (the so called Hutchinson operator) $S(A)=\bigcup_{F \in \mathcal{F}} F(A)$. It is well-known that there is a unique non-empty compact set $K$ such that $S(K)=K$. This set is called the attractor of $\mathcal{F}$. Attractors of ISS's are often termed self-similar sets. The ISS $\mathcal{F}$ and its attractor $K$ are said to satisfy

- SSP (Strong Separation Property) if $F_{i} K \cap F_{j} K=\emptyset$ whenever $i<j<k$,

- SOSC (Strong Open Set Condition) if there is a nonempty open set $U \subseteq X$ that meets $K$ and such that $S(U) \subseteq U$ and $F_{i} U \cap F_{j} U=\emptyset$ whenever $i<j<k$.

SSP is rather restrictive. Obviously SSP implies SOSC, but not vice versa. Nevertheless, Proposition 3.4 infra says that, in a sense, SOSC sets can be approximated from within by SSP sets. Recall that dropping "that meets $K$ " from the definition of SOSC one gets the famous Open Set Condition that is known to be, in Euclidean spaces, equivalent to SOSC (Schief [33]).

For each $p \in k^{<\omega}$ set $F_{p}=F_{p(0)} \circ F_{p(1)} \circ \cdots \circ F_{p(|p|-1)}, K_{p}=F_{p}(K)$. Note that diam $K_{p}=\chi(p)$ diam $K$, where $\chi(p)$ is defined by (2). If $\mathbb{C}(\boldsymbol{r})$ is a Cantor cube, then the ISS given by $F_{i}(f)=i * f$ turns $\mathbb{C}(\boldsymbol{r})$ into a SSP selfsimilar set. On the other hand, it is easy to show that if $K$ satisfies SSP, then 
there are constants $c_{1}$ and $c_{2}$ such that $c_{1} \chi(p) \leqslant \operatorname{dist}\left(K_{i * p}, K_{j * p}\right) \leqslant c_{2} \chi(p)$ for any $p \in k^{<\omega}$ and $i<j<k$. This fact can be phrased as follows.

Lemma 3.2. Every self-similar set satisfying SSP is bi-Lipschitz equivalent to the Cantor cube $\mathbb{C}(\boldsymbol{r})$, where $\boldsymbol{r}$ is the vector of similarity ratios of the underlying ISS.

Lemma 3.3. Every self-similar set is a Lipschitz image of the Cantor cube $\mathbb{C}(\boldsymbol{r})$, where $\boldsymbol{r}$ is the vector of similarity ratios of the underlying ISS.

Proof. If $p \subseteq q \in k^{<\omega}$, then $K_{p} \supseteq K_{q}$. Hence for each $f \in k^{\omega}$ there is a unique $x_{f} \in \bigcap_{n \in \omega} K_{f \backslash n}$; and each $x \in K$ equals to some $x_{f}$. Hence the mapping defined by $\phi(f)=x_{f}$ maps $\mathbb{C}(\boldsymbol{r})$ onto $K$. It is Lipschitz, because

$$
d\left(x_{f}, x_{g}\right) \leqslant \operatorname{diam} K_{f \wedge g} \leqslant \chi(f \wedge g) \operatorname{diam} K \leqslant \rho_{\boldsymbol{r}}(f, g) \operatorname{diam} K .
$$

The solution $s$ of the Moran's equation (3) is called the similarity dimension of $K$ and denoted $\operatorname{dim}_{\mathrm{S}} K$. Hausdorff and similarity dimensions are related as follows:

- $\mathcal{H}^{\operatorname{dim}_{\mathrm{S}} K} K<\infty$, in particular $\operatorname{dim}_{\mathrm{H}} K \leqslant \operatorname{dim}_{\mathrm{S}} K$ (cf. Lemma 3.3)

- If $K$ satisfies SOSC, then $\operatorname{dim}_{\mathrm{H}} K=\operatorname{dim}_{\mathrm{S}} K$ ([34, Theorem 2.6])

- If $K$ satisfies SSP, then $0<\mathcal{H}^{\operatorname{dims}^{K} K} K<\infty$ (cf. Lemma 3.2)

Proposition 3.4. Let $K$ be a self-similar set satisfying SOSC. For each $\varepsilon>0$ there is a SSP self-similar set $C \subseteq K$ such that $\operatorname{dim}_{H} C>\operatorname{dim}_{\mathbf{H}} K-\varepsilon$.

Proof. Though not explicitly stated, the required set $C$ is actually constructed in the proof of [34, Theorem 2.6].

Mapping a Cantor cube onto its square. The following proposition is one of the core tools for our investigation of small sets.

Proposition 3.5. For each Cantor cube $\mathbb{C}$ there are continuous mappings $\pi_{1}, \pi_{2}: \mathbb{C} \rightarrow \mathbb{C}$ such that

(i) $\pi_{1}$ is nearly Lipschitz,

(ii) $h: f \mapsto\left(\pi_{1}(f), \pi_{2}(f)\right)$ is a homeomorphism of $\mathbb{C}$ and $\mathbb{C} \times \mathbb{C}$.

Proof. Let $A \subseteq \omega$ be an infinite set with density 0, i.e.

$$
\lim _{n \rightarrow \infty} \frac{|A \cap n|}{n}=0 .
$$

Let $\phi: \omega \rightarrow \omega \backslash A$ and $\psi: \omega \rightarrow A$ be the unique increasing bijections enumerating $\omega \backslash A$ and $A$. Consider the mappings

$$
\begin{aligned}
& \pi_{1}: \mathbb{C} \rightarrow \mathbb{C}, \quad \pi_{1}(f)=f \circ \phi, \\
& \pi_{2}: \mathbb{C} \rightarrow \mathbb{C}, \quad \pi_{2}(f)=f \circ \psi .
\end{aligned}
$$

and define

$$
h(f)=\left(\pi_{1}(f), \pi_{2}(f)\right)=(f \circ \phi, f \circ \psi), \quad f \in \mathbb{C} .
$$


Both $\pi_{1}$ and $\pi_{2}$ are obviously continuous, and thus so is $h$.

If $f \neq g$, then there is $n \in \omega$ such that $f(n) \neq g(n)$. If $n \notin A$, then $n=\phi(i)$ for some $i \in \omega$ and thus $\pi_{1}(f)(i)=f(n) \neq g(n)=\pi_{1}(g)(n)$ whence $\pi_{1}(f) \neq \pi_{1}(g)$. If $n \in A$, then $\pi_{2}(f) \neq \pi_{2}(g)$ by the same argument. In either case, $h(f) \neq h(g)$. Thus $h$ is one-to-one.

For any $(f, g) \in \mathbb{C} \times \mathbb{C}$ the function

$$
n \mapsto \begin{cases}f \circ \phi^{-1}(n), & n \in \omega \backslash A, \\ g \circ \psi^{-1}(n), & n \in A\end{cases}
$$

is mapped by $h$ on $(f, g)$. Thus $h$ is also onto. Hence $h$ is a homeomorphism.

It remains to prove that $\pi_{1}$ is nearly Lipschitz. To that end we have to show that given any $\varepsilon>0$ there is $\delta>0$ such that

$$
\rho(f \circ \phi, g \circ \phi) \leqslant \rho(f, g)^{1-\varepsilon} \text { whenever } \rho(f, g)<\delta .
$$

Let $\boldsymbol{r} \in(0,1)^{k}$ be such that $\mathbb{C}=\mathbb{C}(\boldsymbol{r})$. Denote $r_{\max }=\max _{i<k} r_{i}$ and $r_{\min }=\min _{i<k} r_{i}$. Choose

- $\eta<\infty$ such that $r_{\max }^{\eta}<r_{\min }$,

- $N \in \omega$ such that $\eta|A \cap n| / n<\varepsilon$ for all $n \geqslant N$,

- $\delta<r_{\min }^{N}$.

Let $f, g \in k^{\omega}$ be such that $\rho(f, g)<\delta$. Put $p=f \wedge g$ and $n=n(f, g)=|p|$. If $\phi(i)<n$, then obviously $f(\phi(i))=g(\phi(i))$. Therefore

$$
\rho(f \circ \phi, g \circ \phi) \leqslant \prod_{i<n, i \notin A} r_{p(i)} \leqslant \frac{\prod_{i<n} r_{p(i)}}{\prod_{i<n, i \in A} r_{p(i)}} \leqslant \frac{\chi(p)}{r_{\min }^{|A \cap n|}} \leqslant \frac{\chi(p)}{r_{\max }^{\eta|A \cap n|}} .
$$

Since $\rho(f, g)<\delta$, we have $n>N$. Since $r_{\min }^{n} \leqslant \chi(p) \leqslant r_{\max }^{n}$,

$$
r_{\max }^{\eta|A \cap n|} \geqslant \chi(p)^{\eta|A \cap n| / n} \geqslant \chi(p)^{\varepsilon}
$$

by the choice of $N$. Therefore (5) yields (4) and $\pi_{1}$ is nearly Lipschitz.

\section{Monotone spaces And Cantor Cubes}

In this section we show that a substantial portion of an analytic metric space satisfying a certain linearity property can be mapped onto any Cantor cube of the same Hausdorff dimension by a nearly Lipschitz map. The property is tailored to fit the proof and is general enough to cover two important classes of spaces: subsets of the line and ultrametric spaces.

Monotone spaces. The following is taken from [30]. Linear orders are considered. We adhere to the usual interval notation $(\leftarrow, a]=\{x \in X$ : $x \leqslant a\},(a, b)=\{x \in X: a<x<b\}$ and likewise $[a, \rightarrow),[a, b]$ etc.

Definition 4.1 ([30]). A metric space $(X, d)$ is called 1-monotone if there is a linear order $<$ on $X$ such that: 
(a) the intervals $(\leftarrow, a],[a, \rightarrow)$ are closed for all $a \in X$,

(b) $d(x, y) \leqslant d(x, z)$ for all $x<y<z$ in $X$.

More generally, $(X, d)$ is called monotone if there is a linear order $<$ on $X$ such that (a) holds and

(c) there is a constant $c$ such that $d(x, y) \leqslant c d(x, z)$ for all $x<y<z$ in $X$.

$X$ is termed $\sigma$-monotone if it is a countable union of monotone subsets.

The line equipped with the Euclidean metric is obviously monotone.

Proposition 4.2 ([30]). A metric space is monotone if and only if it is bi-Lipschitz equivalent to a 1-monotone space.

Proposition 4.3 ([30]). (i) A subspace of a monotone metric space is monotone.

(ii) A metric space with a dense monotone subspace is monotone.

(iii) If $X$ is $\sigma$-monotone, then it is a countable union of closed monotone subspaces.

$\sigma$-monotone spaces have low topological dimension: If $X$ is monotone and separable, then $X$ (topologically) embeds into $\mathbb{R}$ and if $X$ is $\sigma$-monotone, then its topological dimension is at most 1 . But there are spaces with low dimension that are not $\sigma$-monotone: According to [30] there exists a compact set $X \subseteq \mathbb{R}^{2}$ homeomorphic to $[0,1]$ that is not $\sigma$-monotone; in fact, each monotone subset of $X$ is nowhere dense in $X$. However this example is not null-dimensional (recall that a topological space is null-dimensional if it has a base consisting of clopen sets). Question 6.1 and Proposition 6.2 infra show that the following question is relevant to the present context.

Question 4.4. Is every compatible metric on $2^{\omega} \sigma$-monotone?

Recall that a metric space $(X, d)$ is termed ultrametric if the triangle inequality reads

$$
d(x, z) \leqslant \max (d(x, y), d(y, z)) .
$$

Cantor cubes are obviously ultrametric spaces.

Proposition 4.5 ([30]). Each ultrametric space is monotone. In particular, each Cantor cube and each self-similar set satisfying SSP is monotone.

Corollary 4.6. If $X$ is a SOSC self-similar set and $0 \leqslant s<\operatorname{dim}_{\mathrm{H}} X$, then there is a closed monotone set $E \subseteq X$ such that $\operatorname{dim}_{\mathrm{H}} E=s$.

Proof. By Theorem 3.4 there is a SSP set $C \subseteq X$ such that $\operatorname{dim}_{H} C>s$. Davies Theorem yields a compact set $E \subseteq C$ such that $\operatorname{dim}_{\mathrm{H}} E=s$. By Lemma 3.2 and the above proposition, $E$ is the required set. 
Monotone spaces vs. Cantor cubes. We now show that an analytic $\sigma$-monotone space contains a nearly Lipschitz preimage of a Cantor cube.

Theorem 4.7. Let $X$ be an analytic $\sigma$-monotone metric space and $s>0$ such that $\mathcal{H}^{s}(X)>0$. If $\mathbb{C}$ is a Cantor cube with $\operatorname{dim}_{\mathrm{H}} \mathbb{C} \leqslant s$, then there is a compact set $C \subseteq X$ such that $\mathcal{H}^{s}(C)>0$ and a nearly Lipschitz onto mapping $\phi: C \rightarrow \mathbb{C}$.

Proof. Propositions 4.3 and 4.2 ensure that $X$ may be assumed to be 1 monotone. Observe that if $E \subseteq X$ is compact, then $\max E$ and min $E$ exist and belong to $E$. Indeed, the set $\bigcap_{x \in E} E \cap[x, \rightarrow)$, being an intersection of a directed family of compact sets, is nonempty. Its (unique) element is the maximum of $E$; and likewise for the minimum.

We may, and shall, assume that $s=\operatorname{dim}_{\mathrm{H}} \mathbb{C}$.

According to Davies Theorem we may also assume that $X$ is compact. In particular, there are $a, b \in X$ such that $X=[a, b]$.

Frostman's Lemma yields a nontrivial finite Borel measure $\mu \leqslant \mathcal{H}^{s}$ on $X$ such that

$$
\mu B(x, r) \leqslant r^{s} \quad \text { for each } x \in X \text { and } r>0 .
$$

We show that $\mu$ is Darboux in the following sense: For each $E \subseteq X$ compact,

$$
\text { if } 0 \leqslant t \leqslant \mu(E) \text {, then } \mu([a, x] \cap E)=t \text { for some } x \in X \text {. }
$$

Notice that 1-monotonicity of $X$ ensures that $[x, y] \subseteq B(x, d(x, y))$. Therefore the mapping $g(x)=\mu([a, x] \cap E)$ is continuous:

$$
g(y)-g(x) \leqslant \mu[x, y] \leqslant \mu B(x, d(x, y)) \leqslant d(x, y)^{s} .
$$

It follows that the sets

$$
X_{0}=\{x \in X: \mu([a, x] \cap E) \leqslant t\}, \quad X_{1}=\{x \in X: \mu([a, x] \cap E) \geqslant t\}
$$

are closed and hence compact. Let $x_{0}=\max X_{0}, x_{1}=\min X_{1}$. If $x_{1} \leqslant x_{0}$, then $t \leqslant g\left(x_{1}\right) \leqslant g\left(x_{0}\right) \leqslant t$ and we can set $x=x_{1}$. If $x_{1}>x_{0}$, then the interval $\left(x_{0}, x_{1}\right)$ is empty and subadditivity of $\mu$ yields $g\left(x_{1}\right) \leqslant g\left(x_{0}\right)+$ $\mu\left(x_{0}, x_{1}\right] \leqslant t \leqslant g\left(x_{1}\right)$ and we can set $x=x_{1}$, too. (7) is proved.

Let $\boldsymbol{r}=\left\langle r_{0}, r_{1}, \ldots, r_{k-1}\right\rangle \in(0,1)^{k}$ be the parameters of the Cantor cube $\mathbb{C}=\mathbb{C}(\boldsymbol{r})$. Set $r_{\max }=\max _{i<k} r_{i}$ and $r_{\min }=\min _{i<k} r_{i}$. Since $r_{\max }^{s}<1$, there is a sequence $\gamma_{n} \searrow 0$ of positive numbers such that $\sum_{n=1}^{\infty}\left(r_{\max }^{s}\right)^{n \gamma_{n}}<\mu(X)$. Put

$$
\beta=\mu(X)-\sum_{n=1}^{\infty}\left(r_{\max }^{s}\right)^{n \gamma_{n}},
$$

so that $\beta>0$. (By choosing $\left\{\gamma_{n}\right\}$ appropriately, we can make $\beta$ as close to $\mu(X)$ as we wish.)

For each $p \in k^{<\omega}$ put

$$
\varepsilon_{p}=\chi(p)^{1+\gamma_{|p|}}
$$


For each $p \in k^{<\omega}$, we construct inductively a compact set $E_{p} \subset X$ as follows. Put $E_{\emptyset}=X$. Now suppose $E_{p}$ has been constructed. Recalling Moran's equation (3) and using (7), choose

$$
a \leqslant t_{p * 0}<t_{p * 1}<\cdots<t_{p * k} \leqslant b \in X
$$

so that

$$
\mu\left(E_{p} \cap\left[t_{p * i}, t_{p *(i+1)}\right]\right)=r_{i}^{s} \mu E_{p} \quad \text { for } i<k
$$

and define for $i<k$,

$$
E_{p * i}=\left\{x \in E_{p} \cap\left[t_{p * i}, t_{p *(i+1)}\right]: d\left(x, t_{p *(i+1)}\right) \geqslant \varepsilon_{p * i}\right\} .
$$

Note that

$$
E_{p * i} \supseteq E_{p} \cap\left[t_{p * i}, t_{p *(i+1)}\right] \backslash B\left(t_{p *(i+1)}, \varepsilon_{p * i}\right)
$$

and therefore (6) yields

$$
\mu\left(E_{p * i}\right) \geqslant r_{i}^{s} \mu E_{p}-\varepsilon_{p * i}^{s}=r_{i}^{s} \mu E_{p}-\chi(p * i)^{s\left(1+\gamma_{|p * i|}\right)} .
$$

We prove by induction that

$$
\mu(X) \chi(p)^{s} \geqslant \mu\left(E_{p}\right) \geqslant \chi(p)^{s}\left(\mu(X)-\sum_{n=1}^{|p|}\left(r_{\max }^{s}\right)^{n \gamma_{n}}\right)
$$

holds for all $p \in k^{<\omega}$. The left hand inequality is obvious. We prove the right hand one. For $p=\emptyset$ it is trivial. Assume that it holds for $p$ and let $i<k$. Put $m=|p|$. By virtue of $(9)$

$$
\begin{aligned}
\mu E_{p * i} & \geqslant r_{i}^{s} \mu E_{p}-\chi(p * i)^{s\left(1+\gamma_{|p * i|}\right)} \\
& \geqslant r_{i}^{s}\left(\chi(p)^{s} \mu X-\chi(p)^{s} \sum_{n=1}^{m}\left(r_{\max }^{s}\right)^{n \gamma_{n}}\right)-\chi(p * i)^{s} \chi(p * i)^{s \gamma_{m+1}} \\
& \geqslant \chi(p * i)^{s} \mu X-\chi(p * i)^{s}\left(\sum_{n=1}^{m}\left(r_{\max }^{s}\right)^{n \gamma_{n}}+r_{\max }^{(m+1) s \gamma_{m+1}}\right) \\
& =\chi(p * i)^{s}\left(\mu X-\sum_{n=1}^{m+1}\left(r_{\max }^{s}\right)^{n \gamma_{n}}\right) .
\end{aligned}
$$

The induction step is complete and (10) follows. The definition of $\beta$ thus gives

$$
\mu(X) \chi(p)^{s} \geqslant \mu\left(E_{p}\right) \geqslant \beta \chi(p)^{s}
$$

for all $p \in k^{<\omega}$. Thus $\mu E_{p}>0$, and in particular $E_{p} \neq \emptyset$. All $E_{p}$ are obviously compact and $E_{p} \subseteq E_{q}$ whenever $p$ extends $q$. Hence the set

$$
C_{f}=\bigcap_{n \in \omega} E_{f i n}
$$


is nonempty for each $f \in k^{\omega}$. Put

$$
C=\bigcup_{f \in k^{\omega}} C_{f}=\bigcap_{n \in \omega} \bigcup_{p \in k^{n}} E_{p} .
$$

We estimate $\mu(C)$. Moran's equation for $\mathbb{C}$ gives $\sum_{p \in k^{n}} \chi(p)^{s}=1$ for each $n \in \omega$. Hence (11) yields

$$
\mu(C)=\lim _{n \rightarrow \infty} \sum_{p \in k^{n}} \mu\left(E_{p}\right) \geqslant \lim _{n \rightarrow \infty} \beta \sum_{p \in k^{n}} \chi(p)^{s} \geqslant \beta>0
$$

and $\mathcal{H}^{s}(C)>0$ follows, since $\mu \leqslant \mathcal{H}^{s}$.

Define $\phi: C \rightarrow k^{\omega}$ by $\phi(x)=f$ iff $x \in C_{f}$. It is obviously surjective. Provide $k^{\omega}$ with the corresponding metric $\rho=\rho_{\boldsymbol{r}}$. It remains to show that $\phi: C \rightarrow\left(k^{\omega}, \rho\right)$ is nearly Lipschitz. Let $\varepsilon>0$ be given. Since $\gamma_{n} \searrow 0$, there is $n \in \omega$ such that $1-\varepsilon<\frac{1}{1+\gamma_{n}}$. Put $\delta=\min _{|p|<n} \varepsilon_{p}$.

Assume that $x<y$ in $C$ are such that $d(x, y)<\delta$. If $\phi(x)=\phi(y)$, there is nothing to prove. Otherwise there is $p \in k^{<\omega}$ and $i<j<k$ such that $x \in E_{p * i}$ and $y \in E_{p * j}$. Here the assumption that $X$ is 1 -monotone comes in play again: Since $i<j$, we have $x<t_{p *(i+1)} \leqslant y$ and $d\left(x, t_{p *(i+1)}\right) \geqslant \varepsilon_{p * i}$. Therefore $d(x, y) \geqslant \varepsilon_{p * i}$ and thus $\delta>\varepsilon_{p * i}$. The definition of $\delta$ hence yields $|p * i| \geqslant n$. Therefore

$$
d(x, y) \geqslant \varepsilon_{p * i} \geqslant \chi(p * i)^{1+\gamma_{n}} \geqslant \chi(p)^{1+\gamma_{n}} r_{\min }^{1+\gamma_{n}} .
$$

Since $\rho(\phi x, \phi y)=\chi(p)$, this inequality gives

$$
\rho(\phi x, \phi y)=\chi(p) \leqslant \frac{1}{r_{\min }} d(x, y)^{1 /\left(1+\gamma_{n}\right)} \leqslant \frac{1}{r_{\min }} d(x, y)^{1-\varepsilon},
$$

which is enough.

Lemma 3.3 yields the following corollary.

Corollary 4.8. Let $X$ be an analytic $\sigma$-monotone metric space and $K a$ self-similar set. If $\mathcal{H}^{\operatorname{dims}_{\mathrm{S}} K}(X)>0$, then there is a compact set $C \subseteq X$ such that $\mathcal{H}^{\operatorname{dims}_{\mathrm{S}} K}(C)>0$ and a nearly Lipschitz onto mapping $\phi: C \rightarrow K$.

In particular, if $\mathbb{C}_{1}, \mathbb{C}_{2}$ are two Cantor cubes of the same dimension $s$, then there is, for any $\varepsilon>0$, a compact set $C \subseteq \mathbb{C}_{1}$ such that $\mathcal{H}^{s}\left(\mathbb{C}_{1} \backslash C\right)<\varepsilon$ and a continuous mapping $\phi: \mathbb{C}_{1} \rightarrow \mathbb{C}_{2}$ onto $\mathbb{C}_{2}$ such that $\phi\lceil C$ is nearly Lipschitz. This raises a question: If $\mathbb{C}_{1}, \mathbb{C}_{2}$ are two Cantor cubes of the same dimension, is there a nearly Lipschitz bijection $f: \mathbb{C}_{1} \rightarrow \mathbb{C}_{2}$ with a nearly Lipschitz inverse? (Falconer and Marsh [9], see also [10], show that Cantor cubes of the same dimension need not be bi-Lipschitz equivalent.)

Replacing $\mathcal{H}^{s}(X)>0$ with $\operatorname{dim}_{\mathrm{H}} \mathbb{C}<\operatorname{dim}_{\mathrm{H}} X$ yields a stronger conclusion: 
Theorem 4.9. Let $X$ be an analytic $\sigma$-monotone metric space and $\mathbb{C}$ a Cantor cube. If $\operatorname{dim}_{\mathrm{H}} \mathbb{C}<\operatorname{dim}_{\mathrm{H}} X$, then there is a compact set $C \subseteq X$ and a Lipschitz onto mapping $\phi: C \rightarrow \mathbb{C}$.

Proof. We briefly show how to modify the above proof. Let $\mathbb{C}=\mathbb{C}(\boldsymbol{r})$ and let $\operatorname{dim}_{\mathrm{H}} \mathbb{C}=u<s<\operatorname{dim}_{\mathrm{H}} X$. Mutatis mutandis we may assume that $X=[a, b]$ is compact and $\mathcal{H}^{s}(X)>0$. Let $\mu$ be the measure satisfying (6) and (7). Put $R=r_{\max }^{s-u}$. Then $R<1$ and therefore the series $\sum R^{n}$ is convergent. Thus we can choose $\eta>0$ so that $\eta^{s} \sum_{n=1}^{\infty} R^{n}<\mu X$.

This time put $\varepsilon_{p}=\eta \chi(p)$ and construct $E_{p}$ 's so that (9) reads (note that Moran's equation is now $\sum r_{i}^{u}=1$ )

$$
\mu\left(E_{p * i}\right) \geqslant r_{i}^{u} \mu E_{p}-\eta^{s} \chi(p * i)^{s} .
$$

The replacement for the estimate (10) now reads

$$
\mu\left(E_{p}\right) \geqslant \chi(p)^{u}\left(\mu(X)-\eta^{s} \sum_{n=1}^{|p|} R^{n}\right)
$$

and the replacement for (12) is

$$
d(x, y) \geqslant \eta \chi(p * i) \geqslant \eta r_{\min } \chi(p)=\eta r_{\min } \rho(\phi(x), \phi(y)),
$$

which shows that $\phi$ is Lipschitz.

Corollary 4.10. Let $K_{1}, K_{2}$ be self-similar sets. Let $K_{1}$ satisfy SOSC. If $\operatorname{dim}_{\mathrm{S}} K_{2}<\operatorname{dim}_{\mathrm{S}} K_{1}$, then there is a compact set $C \subseteq K_{1}$ and a Lipschitz onto mapping $\phi: C \rightarrow K_{2}$.

Proof. Since $K_{1}$ satisfies SOSC, $\operatorname{dim}_{\mathrm{S}} K_{1}=\operatorname{dim}_{\mathrm{H}} K_{1}$ and by Corollary 4.6 there is a closed monotone subset $X \subseteq K_{1}$ such that $\operatorname{dim}_{\mathrm{H}} X>\operatorname{dim}_{\mathrm{S}} K_{2}$. Apply Lemma 3.3 and the above theorem.

\section{Small Sets With LARGe Dimension}

Suppose $\mathcal{S}$ is some class of separable metric spaces. Elements of $\mathcal{S}$ are thought of as "small sets". We will consider the following closure properties of $\mathcal{S}$.

- $\mathcal{S}$ is backwards closed. That means that $\mathcal{S}$ is closed under one-to-one continuous preimages, i.e. if $f: X \rightarrow Y$ is one-to-one continuous and $Y \in \mathcal{S}$, then $X \in \mathcal{S}$. Note that if $\mathcal{S}$ is backwards closed, then it is closed under homeomorphisms and embeddings.

- $\mathcal{S}$ is strongly backwards closed. That means that $\mathcal{S}$ is closed under $\mathcal{S}$-toone continuous preimages, i.e. if $f: X \rightarrow Y$ is continuous, $f^{-1}(y) \in \mathcal{S}$ for all $y \in Y$ and $Y \in \mathcal{S}$, then $X \in \mathcal{S}$.

- $\mathcal{S}$ is $\sigma$-additive, i.e. if $X$ is a union of countably many elements of $\mathcal{S}$, then $X \in \mathcal{S}$. 
Cardinal invariants. The following are some of the most common ideals in topology, set theory and measure theory.

- Let $\mu$ denote the standard product measure on $2^{\omega}$, and $\mathcal{N}$ the $\sigma$-ideal of $\mu$-negligible subsets of $2^{\omega}$.

- $\mathcal{M}$ is the $\sigma$-ideal of meager subsets of $2^{\omega}$.

- $\mathcal{E}$ is the $\sigma$-ideal generated by $\mu$-negligible closed subsets of $2^{\omega}$.

For any ideal $\mathcal{J}$ denote non $\mathcal{J}=\min \{|A|: A \notin \mathcal{J}\}$.

It is well-known that either of the three relations of non $\mathcal{N}$ and non $\mathcal{M}$ is consistent with ZFC. It is obvious that non $\mathcal{E} \leqslant \min (\operatorname{non} \mathcal{N}$, non $\mathcal{M})$. It is also known that both non $\mathcal{E}=\min (\operatorname{non} \mathcal{N}, \operatorname{non} \mathcal{M})$ and $\operatorname{non} \mathcal{E}<\min (\operatorname{non} \mathcal{N}$, non $\mathcal{M})$ are consistent, see [2].

The $\mathcal{N}$ part of the following fact is well-known and follows at once from the Isomorphism Theorem for Measures ([21, (17.41)]).

Lemma 5.1. Let $\nu$ be a nontrivial finite diffused Borel measure on $2^{\omega}$ and $\mathcal{N}(\nu), \mathcal{E}(\nu)$, respectively, the $\sigma$-ideals generated by $\nu$-negligible sets and $\nu$ negligible $F_{\sigma}$-sets. Then non $\mathcal{N}(\nu)=\operatorname{non} \mathcal{N}$ and non $\mathcal{E}(\nu)=$ non $\mathcal{E}$.

Proof. We outline the proof of the $\mathcal{E}$ part. Assume without loss of generality that $\nu$ is a diffused probability Borel measure on a compact set $S \subseteq[0,1]$ that is strictly positive (i.e. $\nu(U)>0$ for each nonempty set $U$ open in $S$ ). For $x \in S$ set $g(x)=\nu([0, x])$. The mapping $g: S \rightarrow[0,1]$ is obviously continuous and onto. Denoting $\lambda$ the Lebesgue measure on $[0,1]$, it is also obvious that $E \in \mathcal{E}(\nu) \Leftrightarrow g(E) \in \mathcal{E}(\lambda)$ holds for any set $E \subseteq S$. Since $g$ is two-to-one, this is enough for non $\mathcal{E}(\nu)=\operatorname{non} \mathcal{E}(\lambda)$. In particular, non $\mathcal{E}=$ non $\mathcal{E}(\lambda)$, and non $\mathcal{E}(\nu)=$ non $\mathcal{E}$ follows.

Small sets in $\sigma$-monotone spaces. The goal of this subsection is to show that if $\mathcal{S}$ is backwards closed, then the existence of a set in $\mathcal{S}$ with large cardinality implies that many sets in $\mathcal{S}$ have large Hausdorff or upper Hausdorff dimension.

In the sequel we often employ cardinal hypotheses on $\mathcal{S}$ : Given a cardinal $\kappa($ in most cases $\kappa=$ non $\mathcal{N}$ or $\kappa=$ non $\mathcal{E}$ ), define

$$
\kappa \prec \mathcal{S} \stackrel{\text { def }}{\equiv} \text { there is } S \in \mathcal{S} \text { such that }|S|=\kappa .
$$

Note that if $\mathcal{S}$ is backwards closed and $\kappa \prec \mathcal{S}$, then the witnessing space can be actually assumed to be a subset of $2^{\omega}$ : For any separable metric space embeds into $[0,1]^{\omega}$ and the latter is a continuous image of $2^{\omega}$.

We first prove the result for Cantor cubes.

Lemma 5.2 (Cantor Cube Lemma). Let $\mathcal{S}$ be backwards closed and $\mathbb{C}$ a Cantor cube.

(i) If non $\mathcal{N} \prec \mathcal{S}$, then $\mathbb{C}$ contains a set $E \in \mathcal{S}$ such that $\operatorname{dim}_{\mathrm{H}} E=\operatorname{dim}_{\mathrm{H}} \mathbb{C}$.

(ii) If non $\mathcal{E} \prec \mathcal{S}$, then $\mathbb{C}$ contains a set $E \in \mathcal{S}$ such that $\overline{\operatorname{dim}}_{\mathrm{H}} E=\operatorname{dim}_{\mathrm{H}} \mathbb{C}$. 
Proof. We prove only (i), since (ii) is proved in the same manner. Let $s=\operatorname{dim}_{\boldsymbol{H}} \mathbb{C}$. Recall that $\mathcal{H}^{s}(\mathbb{C})=1$, so that $\mathcal{H}^{s}$ is a finite Borel measure on $\mathbb{C}$. Hence Lemma 5.1 yields a set $A \subseteq \mathbb{C}$ such that $\mathcal{H}^{s}(A)>0$ and $|A|=$ non $\mathcal{N}$. Since $\mathcal{S}$ is backwards closed and $\mathbb{C}$ is homeomorphic to $2^{\omega}$, there is $B \in \mathcal{P}(\mathbb{C}) \cap \mathcal{S}$ such that $|B|=$ non $\mathcal{N}$. Enumerate the two sets $A=$ $\left\{a_{\alpha}: \alpha<\operatorname{non} \mathcal{N}\right\}, B=\left\{b_{\alpha}: \alpha<\right.$ non $\left.\mathcal{N}\right\}$. Consider the diagonal set $D=$ $\left\{\left(a_{\alpha}, b_{\alpha}\right): \alpha<\operatorname{non} \mathcal{N}\right\}$ and the mappings $\pi_{1}, \pi_{2}$ and $h$ of Proposition 3.5. For each $\alpha<\operatorname{non} \mathcal{N}$ set $e_{\alpha}=h^{-1}\left(a_{\alpha}, b_{\alpha}\right)$ and put

$$
E=\left\{e_{\alpha}: \alpha<\operatorname{non} \mathcal{N}\right\}=h^{-1}(D) .
$$

Obviously $\pi_{1}\left(e_{\alpha}\right)=a_{\alpha}$ and $\pi_{2}\left(e_{\alpha}\right)=b_{\alpha}$. Therefore $\pi_{1}$ takes $E$ onto $A$ and $\pi_{2}$ takes $E$ onto $B$. The mapping $\pi_{2}$ is one-to-one on $E$ and $B \in \mathcal{S}$. Hence $E \in \mathcal{S}$ as well. On the other hand, since $\pi_{1}$ is nearly Lipschitz, Lemma 2.6 ensures that $\operatorname{dim}_{\mathrm{H}} E \geqslant \operatorname{dim}_{\mathrm{H}} \pi_{1} E=\operatorname{dim}_{\mathrm{H}} A=s=\operatorname{dim}_{\mathrm{H}} \mathbb{C}$, as required.

The main theorem of this section follows from the Cantor Cube Lemma and Theorem 4.7 by use of the following easy lemma.

Lemma 5.3 (Preimage Lemma). Let $\mathcal{S}$ be backwards closed. Let $X, Y$ be metric spaces, $\phi: X \rightarrow Y$ a nearly Lipschitz mapping onto $Y$ and $D \in \mathcal{S}$ a subset of $Y$. There is a subset $E \in \mathcal{S}$ of $X$ such that $\operatorname{dim}_{\mathrm{H}} E \geqslant \operatorname{dim}_{\mathrm{H}} D$ and $\overline{\operatorname{dim}}_{\mathrm{H}} E \geqslant \overline{\operatorname{dim}}_{\mathrm{H}} D$.

Proof. For each $y \in D$, pick $\widetilde{y} \in \phi^{-1}(y)$ and set $E=\{\widetilde{y}: y \in D\}$. Then $\phi: E \rightarrow D$ is one-to-one, onto and nearly Lipschitz. Hence $E \in \mathcal{S}$ and the remainder of the lemma follows directly from Lemma 2.6.

Theorem 5.4. Let $\mathcal{S}$ be backwards closed. Let $X$ be an analytic $\sigma$-monotone space and $s>0$ such that $\mathcal{H}^{s}(X)>0$.

(i) If non $\mathcal{N} \prec \mathcal{S}$, then $X$ contains a set $E \in \mathcal{S}$ such that $\operatorname{dim}_{\mathrm{H}} E=s$.

(ii) If non $\mathcal{E} \prec \mathcal{S}$, then $X$ contains a set $E \in \mathcal{S}$ such that $\overline{\operatorname{dim}}_{\mathrm{H}} E=s$.

Proof. We prove only (i), since (ii) is proved in the same manner. By Theorem 4.7 there is a compact set $C \subseteq X$ and a nearly Lipschitz map $\phi: C \rightarrow \mathbb{C}$ onto any Cantor cube for which $\operatorname{dim}_{H} \mathbb{C}=s$. By the Cantor Cube Lemma there is a set $D \in \mathcal{P}(\mathbb{C}) \cap \mathcal{S}$ such that $\operatorname{dim}_{\mathrm{H}} D=s$. Finally, by the Preimage Lemma there is a set $E \in \mathcal{P}(X) \cap \mathcal{S}$ such that $\operatorname{dim}_{\mathrm{H}} D=s$.

Corollary 5.5. Let $\mathcal{S}$ be backwards closed and $\sigma$-additive. Let $X$ be an analytic $\sigma$-monotone space.

(i) If non $\mathcal{N} \prec \mathcal{S}$, then $X$ contains a set $E \in \mathcal{S}$ such that $\operatorname{dim}_{\mathrm{H}} E=$ $\operatorname{dim}_{\mathrm{H}} X$.

(ii) If non $\mathcal{E} \prec \mathcal{S}$, then $X$ contains a set $E \in \mathcal{S}$ such that $\overline{\operatorname{dim}}_{\mathrm{H}} E=$ $\operatorname{dim}_{H} X$. 
Proof. Choose a sequence $s_{n} \nearrow \operatorname{dim}_{\mathrm{H}} X$. Using Theorem 5.4 for each $n$, we can find $E_{n} \in \mathcal{P}(X) \cap \mathcal{S}$ for which $\operatorname{dim}_{\mathrm{H}} E_{n} \geqslant s_{n}\left(\overline{\operatorname{dim}}_{\mathrm{H}} E_{n} \geqslant s_{n}\right.$, respectively). Now let $E=\bigcup_{n} E_{n}$ and appeal to $\sigma$-additivity of $\mathcal{S}$ to conclude $E \in \mathcal{S}$.

Varying the closure and cardinal hypotheses yields a number of corollaries. Here is a sample one that follows from Proposition 4.5 and Corollary 4.6.

Corollary 5.6. Let $\mathcal{S}$ be backwards closed and $\sigma$-additive and suppose non $\mathcal{N} \prec \mathcal{S}$. Then:

(i) Each analytic ultrametric space $X$ contains a set $E \in \mathcal{S}$ for which $\operatorname{dim}_{\mathrm{H}} E=\operatorname{dim}_{\mathrm{H}} X$.

(ii) If $X$ is a SOSC self-similar set and $0 \leqslant s \leqslant \operatorname{dim}_{\mathrm{H}} X$, then $X$ contains a set $E \in \mathcal{S}$ for which $\operatorname{dim}_{\mathrm{H}} E=s$.

Small sets in Euclidean spaces. The preceding results certainly apply to analytic subsets of the line. To extend them to higher dimensions, we use classical projection and intersection theorems. Let $n \in \omega$ and let $V$ be a linear subspace of $\mathbb{R}^{n}$. Denote the orthogonal complement of $V$ by $V^{\perp}$ and the orthogonal projection onto $V$ by $\operatorname{proj}_{V}$. If $x \in \mathbb{R}^{n}$, then $V+x$ denotes the shift of $V$ by $x$, i.e. the unique affine copy of $V$ parallel to $V$ passing through $x$.

Let $m \in \omega, m<n$. The symbol $G(n, m)$ denotes the Grassmann manifold, which is the space of all $m$-dimensional linear subspaces of $\mathbb{R}^{n}$. The Grassmann measure is the unique uniform Borel probability measure on $G(n, m)$ and is denoted by $\gamma_{n, m}$. We refer to [24] for further details.

Lemma 5.7. Let $X \subseteq \mathbb{R}^{n}$ be compact and $m<s \leqslant n$. If $0<\mathcal{H}^{s}(X)<\infty$, then the set

$$
X_{V}=\left\{x \in V: \operatorname{dim}_{\mathrm{H}} X \cap\left(V^{\perp}+x\right)=s-m\right\}
$$

is Borel and for $\gamma_{n, m}$-almost all $V \in G(n, m), \mathcal{H}^{m}\left(X_{V}\right)>0$.

Proof. This is [24, Theorem 10.10], except for " $X_{V}$ is Borel". But that follows at once from $[25$, Theorem 6.1].

Lemma 5.8 ([24, Corollary 9.4]). Let $X \subseteq \mathbb{R}^{n}$ be Borel. If $\operatorname{dim}_{\mathrm{H}} X \leqslant m$, then $\operatorname{dim}_{\mathrm{H}} \operatorname{proj}_{V}(X)=\operatorname{dim}_{\mathrm{H}} X$ for $\gamma_{n, m}$-almost all $V \in G(n, m)$.

Lemma 5.9 ([12, Corollary 7.12]). Let $E \subseteq \mathbb{R}^{n}$ be arbitrary, $V \in G(n, m)$ and $D \subseteq \operatorname{proj}_{V} E$. Then

$$
\operatorname{dim}_{\mathrm{H}} E \geqslant \operatorname{dim}_{\mathrm{H}} D+\inf _{x \in D} \operatorname{dim}_{\mathrm{H}} E \cap\left(V^{\perp}+x\right) .
$$

We also need the upper Hausdorff dimension counterpart of this lemma. 
Lemma 5.10. Let $E \subseteq \mathbb{R}^{n}$ be arbitrary, $V \in G(n, m)$ and $D \subseteq \operatorname{proj}_{V} E$. Then

$$
\overline{\operatorname{dim}}_{\mathrm{H}} E \geqslant \overline{\operatorname{dim}}_{\mathrm{H}} D+\inf _{x \in D} \overline{\operatorname{dim}}_{\mathrm{H}} E \cap\left(V^{\perp}+x\right) .
$$

Proof. For $x \in V$ and any set $A \subseteq \mathbb{R}^{n}$ write $A^{x}=A \cap\left(V^{\perp}+x\right)$. Let $c<\inf _{x \in D} \overline{\operatorname{dim}}_{\mathrm{H}} E^{x}$ and $d<\overline{\operatorname{dim}}_{\mathrm{H}} D$. It is enough to show that $\overline{\operatorname{dim}}_{\mathrm{H}} E \leqslant$ $c+d$, so, aiming for a contradiction, assume the contrary. Then there is a countable family of compact sets $\left\{K_{n}\right\}$ such that $E \subseteq \bigcup_{n} K_{n}$ and

$$
\operatorname{dim}_{\mathrm{H}} K_{n}<c+d, \quad n \in \omega .
$$

Fix $x \in D$. Since $E^{x} \subseteq \bigcup_{n} K_{n}^{x}$, there is $n$ such that $\operatorname{dim}_{\mathrm{H}} K_{n}^{x}>c$, which in turn implies $\delta>0$ such that $\mathcal{H}_{\delta}^{c}\left(K_{n}^{x}\right) \geqslant 1$ (cf. (1)). Therefore

$$
D=\bigcup_{n \in \omega, \delta>0}\left\{x \in D: \mathcal{H}_{\delta}^{c}\left(K_{n}^{x}\right) \geqslant 1\right\}
$$

In particular there is $n$ and $\delta>0$ such that

$$
\overline{\operatorname{dim}}_{\mathbf{H}}\left\{x \in D: \mathcal{H}_{\delta}^{c}\left(K_{n}^{x}\right) \geqslant 1\right\}>d .
$$

The beginning of the proof of [25, Theorem 6.1] shows that the mapping $x \mapsto \mathcal{H}_{\delta}^{c}\left(K_{n}^{x}\right)$ is upper semicontinuous on $V$. Therefore

$$
C=\left\{x \in V: \mathcal{H}_{\delta}^{c}\left(K_{n}^{x}\right) \geqslant 1\right\}
$$

is closed. It is also bounded, for $K_{n}$ is compact. Hence $C$ is a compact set. Therefore (14) yields $\operatorname{dim}_{\mathrm{H}} C>d$ and (15) yields $\operatorname{dim}_{\mathrm{H}} K_{n}^{x} \geqslant c$ for all $x \in C$. Finally, apply Lemma 5.9 to get $\operatorname{dim}_{\mathrm{H}} K_{n} \geqslant c+d$, which contradicts (13).

Theorem 5.11. Let $\mathcal{S}$ be strongly backwards closed and $\sigma$-additive. Let $X \subseteq \mathbb{R}^{n}$ be analytic.

(i) If non $\mathcal{N} \prec \mathcal{S}$, then $X$ contains a set $E \in \mathcal{S}$ such that $\operatorname{dim}_{\mathrm{H}} E=$ $\operatorname{dim}_{\mathrm{H}} X$.

(ii) If non $\mathcal{E} \prec \mathcal{S}$, then $X$ contains a set $E \in \mathcal{S}$ such that $\overline{\operatorname{dim}}_{\mathrm{H}} E=$ $\operatorname{dim}_{\mathrm{H}} X$.

Proof. (i) Let $n \in \omega$. For $n=1$ the assertion follows at once from Corollary 5.5. We proceed by induction. Assume that $n>1$ and that the assertion holds for $n-1$. Let $X \subseteq \mathbb{R}^{n}$ be a Borel set and let $s=\operatorname{dim}_{\mathrm{H}} X$.

If $s \leqslant n-1$, then there is, by Lemma 5.8, a hyperplane $V \in G(n, n-1)$ such that $\operatorname{dim}_{\mathrm{H}} \operatorname{proj}_{V} X=s$. The induction hypothesis yields a set $A \subseteq$ $\operatorname{proj}_{V} X, A \in \mathcal{S}$, such that $\operatorname{dim}_{\mathrm{H}} A=s$. Since $\operatorname{proj}_{V}$ is Lipschitz, the Preimage Lemma yields a set $E \in \mathcal{P}(X) \cap \mathcal{S}$ such that $\operatorname{dim}_{\mathrm{H}} E=s$.

Now suppose $s>n-1$. Since $\mathcal{S}$ is $\sigma$-additive, we may assume that $\mathcal{H}^{s}(X)>0$. Passing to the subset whose existence is guaranteed by Davies 
Theorem, we may assume that $X$ is compact and $0<\mathcal{H}^{s}(X)<\infty$. From Lemma 5.7 (with $m=1$ ), there is a line $L \in G(n, 1)$ such that the set

$$
X_{L}=\left\{x \in L: \operatorname{dim}_{\mathrm{H}} X \cap\left(L^{\perp}+x\right)=s-1\right\}
$$

is Borel and satisfies $\mathcal{H}^{1}\left(X_{L}\right)>0$. Now apply the induction hypothesis to obtain, firstly, a set $D \subseteq X_{L}, D \in \mathcal{S}$ with $\operatorname{dim}_{\mathrm{H}} D=1$ and, secondly, for each $x \in D$, a set $E_{x} \subseteq X \cap\left(L^{\perp}+x\right)$ with $E_{x} \in \mathcal{S}$ and $\operatorname{dim}_{\mathrm{H}} E_{x}=s-1$. Put

$$
E=\bigcup_{x \in D} E_{x}
$$

so that the sections of $E$ perpendicular to $V$ are precisely the $E_{x}$ 's and thus have Hausdorff dimensions $s-1$. By Lemma 5.9, the set $E$ satisfies $\operatorname{dim}_{\mathrm{H}} E \geqslant(s-1)+\operatorname{dim}_{\mathrm{H}} D=s$. Consider the restriction $\pi=\operatorname{proj}_{L} \uparrow E$ of the projection. It is Lipschitz and takes $E$ onto a set $D \in \mathcal{S}$. Moreover $\pi^{-1}(x)=E_{x} \in \mathcal{S}$ for all $x \in D$. Consequently $E \in \mathcal{S}$ : for $\mathcal{S}$ is strongly backwards closed. The induction step is complete, and so is the proof (i).

(ii) is proved exactly the same way, except that Lemma 5.9 has to be replaced by Lemma 5.10.

I do not know if "strongly backwards closed" can be relaxed to "backwards closed" in the preceding theorem.

\section{General metric spaces}

The methods used to get a small subset of large Hausdorff dimension apply only to special classes of metric spaces. In particular I do not know the answer to the following question:

Question 6.1. Let $\mathcal{S}$ be backwards closed and $\sigma$-additive and non $\mathcal{N} \prec \mathcal{S}$. Given a compact metric space $X$, is there a set $E \subseteq X$ with $E \in \mathcal{S}$ and $\operatorname{dim}_{\mathrm{H}} E=\operatorname{dim}_{\mathrm{H}} X$ ?

Note that by virtue of Davies Theorem, relaxing "compact" to "analytic" does not affect the answer. Proposition 8.2 below shows that separability of $X$ alone is not enough for an affirmative answer.

The following proposition shows the relevance of Question 4.4: An affirmative answer yields an affirmative answer to Question 6.1.

Proposition 6.2. Let $\mathcal{S}$ be backwards closed, $\sigma$-additive and suppose non $\mathcal{N} \prec$ $\mathcal{S}$. Let $X$ be an analytic metric space such that each null-dimensional compact subset of $X$ is $\sigma$-monotone. Then $X$ contains a set $E \in \mathcal{S}$ for which $\operatorname{dim}_{\mathrm{H}} E=\operatorname{dim}_{\mathrm{H}} X$.

Proof. It is enough to show that if $\mathcal{H}^{s}(X)>0$, then there is a nulldimensional compact set $K \subseteq X$ such that $\mathcal{H}^{s}(K)>0$, and apply Theorem 5.4 to $K$. First use Davies Theorem to get compact $K_{0} \subseteq X$ with 
$0<\mathcal{H}^{s}\left(K_{0}\right)<\infty$. The restriction of $\mathcal{H}^{s}$ to Borel subsets of $K_{0}$ is a finite Borel measure on $K_{0}$. Therefore [40, 2.8] yields a closed null-dimensional subset $K \subseteq K_{0}$ such that $\mathcal{H}^{s}(K)>0$.

But since we do not know the answer to Question 4.4, there is nothing so far we can conclude about a general analytic metric space. Our next goal is to establish a weaker conclusion that applies to any metric space: There is a small set of Hausdorff dimension no less than the topological dimension of the space.

Topological dimension and Lipschitz maps. The topological dimension we consider is covering dimension and we denote it by $\operatorname{dim} X$. Recall that if $X$ is a metric space, then $\operatorname{dim} X$ equals the large inductive dimension of $X$ (the Katětov-Morita Theorem) and if, in addition, $X$ is separable, then $\operatorname{dim} X$ equals the small inductive dimension of $X$, see [7] or [8]. We shall need the following theorem.

Theorem 6.3 ([41, Lemma 5.1]). Let $X$ be a metric space. If $\operatorname{dim} X \geqslant$ $n \in \omega$, then there is a countable family $\left\{f_{i}: i \in \omega\right\}$ of Lipschitz mappings $f_{i}: X \rightarrow[0,1]^{n}$ such that $\bigcup_{i \in \omega} f_{i}(X)=[0,1]^{n}$.

We digress briefly and discuss some consequences of this theorem. Can one reduce the number of Lipschitz mappings by placing stronger assumptions on $X$ ? Here are two simple results of this kind.

Corollary 6.4. Let $X$ be a $\sigma$-compact metric space. If $\operatorname{dim} X \geqslant n \in \omega$, then there is a Lipschitz mapping that maps $X$ onto $[0,1]^{n}$.

Proof. Start with the countable family of Lipschitz mappings given by the theorem. Since $X$ is $\sigma$-compact, by the Baire Category Theorem, there is $i \in \omega$ such that $f_{i}(X)$ contains a closed nondegenerate cube $I$. The mapping $\pi$ that assigns to each $x \in f_{i}(X)$ the closest point of $I$ is obviously Lipschitz. Therefore $\pi \circ f_{i}$ is Lipschitz and maps $X$ onto $I$, which is enough.

Corollary 6.5. Let $X$ be an analytic metric space. If $\operatorname{dim} X \geqslant 2$, then there is a Lipschitz mapping that maps $X$ onto $[0,1]^{2}$.

Proof. This time $[0,1]^{2}$ is covered by analytic sets $f_{i}(X)$. Hence $\mathcal{H}^{2}\left(f_{i} X\right)>$ 0 for some $i \in \omega$. By a result of David Preiss (see [23]), any analytic set in $\mathbb{R}^{2}$ of positive Lebesgue measure maps onto a disc by a Lipschitz mapping. Now proceed as before.

Theorem 6.3 also yields the following classical result.

Corollary 6.6 ([17]). If $X$ is a metric space and $\operatorname{dim} X \geqslant n$, then $\mathcal{H}^{n}(X)>$ 0. In particular, $\operatorname{dim}_{\mathrm{H}} X \geqslant \operatorname{dim} X$. 
While the classical proof is based on integration, this one requires only the above theorem, which is purely topological by nature (as well as its proof), and the trivial Lemma 2.3:

Proof. $[0,1]^{n}$ is covered by countably many sets $f_{i}(X)$. One of them thus has positive $n$-dimensional Hausdorff measure. Now apply Lemma 2.3.

Small sets of large Hausdorff dimension. We shall need a refinement of the Cantor Cube Lemma 5.2.

Lemma 6.7. Let $\mathcal{S}$ be backwards closed and $\mathbb{C}$ a Cantor cube. If non $\mathcal{N} \prec \mathcal{S}$, then $\mathbb{C}$ contains a set $E \in \mathcal{S}$ such that for any countable cover $\left\{L_{n}: n \in \omega\right\}$ of $\mathbb{C}$ there is $n$ such that $\operatorname{dim}_{H}\left(E \cap L_{n}\right)=\operatorname{dim}_{H} \mathbb{C}$. Similar statement holds for non $\mathcal{E}$ and $\overline{\operatorname{dim}}_{\mathrm{H}}$.

Proof. We use the notation of the Cantor Cube Lemma proof. For each $n$ put $A_{n}=\left\{a_{\alpha}:\left(a_{\alpha}, b_{\alpha}\right) \in h\left(L_{n}\right)\right\}$. Since $\left\{L_{n}\right\}$ covers $\mathbb{C}$, the family $\left\{A_{n}\right\}$ covers $A$. Since $\mathcal{H}^{s}(A)>0$, there is $n$ for which $\mathcal{H}^{s}\left(A_{n}\right)>0$ and a fortiori $\operatorname{dim}_{\mathrm{H}} A_{n}=s$. If $a_{\alpha} \in A_{n}$, then $\left(a_{\alpha}, b_{\alpha}\right) \in h\left(L_{n}\right) \cap D$. Consequently $e_{\alpha}=$ $h^{-1}\left(a_{\alpha}, b_{\alpha}\right) \in h^{-1}\left(h\left(L_{n}\right) \cap D\right)=L_{n} \cap E$ and thus $\pi_{1}\left(e_{\alpha}\right)=a_{\alpha}$. This proves that $A_{n} \subseteq \pi_{1}\left(L_{n} \cap E\right)$. Now apply Lemma 2.6 to get $\operatorname{dim}_{\mathbf{H}}\left(L_{n} \cap E\right)=s$.

Theorem 6.8. Let $\mathcal{S}$ be backwards closed and $X$ a metric space.

(i) If non $\mathcal{N} \prec \mathcal{S}$, then $X$ contains a set $E \in \mathcal{S}$ such that $\operatorname{dim}_{\mathrm{H}} E \geqslant \operatorname{dim} X$.

(ii) If non $\mathcal{E} \prec \mathcal{S}$, then $X$ contains a set $E \in \mathcal{S}$ such that $\overline{\operatorname{dim}}_{\mathrm{H}} E \geqslant \operatorname{dim} X$.

Proof. Let $\operatorname{dim} X \geqslant n \in \omega$.

By Theorem 4.7, there is a compact set $C \subseteq[0,1]$ and a nearly Lipschitz onto mapping $\phi: C \rightarrow \mathbb{C}\left(\frac{1}{2}, \frac{1}{2}\right)$. The $n$-th cartesian power $\phi^{n}$ of $\phi$ is thus a nearly Lipschitz mapping of $C^{n}$ onto $\left(\mathbb{C}\left(\frac{1}{2}, \frac{1}{2}\right)\right)^{n}$ equipped with the maximum metric. The latter power is easily seen to be isometric with $\mathbb{C}(\underbrace{\frac{1}{2}, \frac{1}{2}, \ldots, \frac{1}{2}}_{2^{n} \text { times }})$. Thus, in summary, there is a compact set $K \subseteq[0,1]^{n}$, a Cantor cube $\mathbb{C}$ with $\operatorname{dim}_{H} \mathbb{C}=n$ and a nearly Lipschitz onto mapping $\psi: K \rightarrow \mathbb{C}$.

Now consider the family $\left\{f_{i}: i \in \omega\right\}$ of Lipschitz mappings given by Lemma 6.3 and the corresponding cover $\left\{\psi\left(f_{i}(X) \cap K\right): i \in \omega\right\}$ of $\mathbb{C}$. Then Lemma 6.7 yields an $i \in \omega$ such that $\psi\left(f_{i}(X) \cap K\right)$ contains a set $A \in \mathcal{S}$ for which $\operatorname{dim}_{\mathrm{H}} A=n$. Notice that $\psi \circ f_{i}$ is nearly Lipschitz and apply the Preimage Lemma.

Small sets of large topological dimension. For sake of completeness we recall [41, Theorem 3.6]. In terms of our notation it reads: 
Theorem 6.9. Let $\mathcal{S}$ be backwards closed, $\sigma$-additive and suppose that $\mathfrak{c} \prec$ $\mathcal{S}$. Then each separable metric space $X$ contains a set $E \in \mathcal{S}$ such that $\operatorname{dim} E \geqslant \operatorname{dim} X-1$.

\section{UNIVERSALLY NULL AND UNIVERSALLY MEAGER SETS}

There are two important classes of small sets that satisfy the hypotheses of the preceding theorems, namely universally null and universally meager sets.

Universally null sets. Recall that a metric space $X$ is universally null (UN) if each finite diffused Borel measure $\mu$ on $X$ is trivial, i.e. $\mu(X)=0$. If $E \subseteq X$ is a subspace of $X$, then $E$ is $\mathbf{U N}$ iff it is $\mu$-negligible for each finite Borel diffused measure on $X$. This property is obviously countably additive.

Universally meager sets. Piotr Zakrzewski [37, 36] defines a set $E$ in a metric space to be universally meager (UM) if, for every perfect Polish space $Z$, a subset $Y \subseteq Z$ and a Borel one-to-one mapping $f: Y \rightarrow E, Y$ is meager in $Z$. This is a strengthening of the classical notion of a perfectly meager set, which is a set whose every perfect subset is meager in itself. In his papers, Zakrzewski gathers evidence that the notion of $\mathbf{U M}$ is a category counterpart of $\mathbf{U N}$. As to the relation of $\mathbf{U N}$ and $\mathbf{U M}$, it is not provable within ZFC that either of the two classes is included in the other, but to date there is no ZFC proof that the two classes differ.

The following lemma claims that the classes UN, UM and UN $\cap \mathbf{U M}$ satisfy the closure hypotheses of all of the above theorems on small sets. Most of it is obvious or easy to prove. The only nontrivial part - strongly backwards closeness of UM - follows at once from [36, Lemma 2.1].

Lemma 7.1. The classes $\mathbf{U N}$ and $\mathbf{U M}$ are $\sigma$-additive and strongly backwards closed.

A deep result of Edwin Grzegorek ensures that the three classes satisfy also the cardinal hypotheses. As for UM, Grzegorek proves it for absolutely first category sets in place of $\mathbf{U M}$, but Zakrzewski shows in [37, Theorem 2.1] that the two classes are equal.

Lemma $7.2([15])$. non $\mathcal{N} \prec \mathbf{U N}$ and $\operatorname{non} \mathcal{M} \prec \mathbf{U M}$.

The following folklore lemma follows at once from the Isomorphism Theorem for Measures $([21,(17.41)])$ and the definitions.

Lemma 7.3. Let $E \subseteq 2^{\omega}$. If $|E|<\operatorname{non} \mathcal{N}$, then $E$ is UN. If $|E|<\operatorname{non} \mathcal{M}$, then $E$ is UM.

Corollary 7.4. $\min (\operatorname{non} \mathcal{N}, \operatorname{non} \mathcal{M}) \prec \mathbf{U N} \cap \mathbf{U M}$. 
Proof. Suppose first that non $\mathcal{M}<$ non $\mathcal{N}$. By Lemma 7.2 there is $A \subseteq 2^{\omega}$ UM such that $|A|=\operatorname{non} \mathcal{M}<\operatorname{non} \mathcal{N}$. By Lemma 7.3, $A$ is UN. The same argument works if non $\mathcal{M}>$ non $\mathcal{N}$.

Now suppose that non $\mathcal{M}=$ non $\mathcal{N}$. Take a UN set $A=\left\{a_{\alpha}: \alpha<\right.$ non $\mathcal{N}\} \subseteq 2^{\omega}$ and a $\mathbf{U M}$ set $B=\left\{b_{\alpha}: \alpha<\operatorname{non} \mathcal{N}\right\} \subseteq 2^{\omega}$ and consider the diagonal set $D=\left\{\left(a_{\alpha}, b_{\alpha}\right): \alpha<\right.$ non $\left.\mathcal{N}\right\} \subseteq 2^{\omega} \times 2^{\omega}$. Obviously $|D|=\operatorname{non} \mathcal{N}=\operatorname{non} \mathcal{M}$. Both projections $\left(a_{\alpha}, b_{\alpha}\right) \mapsto a_{\alpha},\left(a_{\alpha}, b_{\alpha}\right) \mapsto b_{\alpha}$ are obviously continuous and one-to-one. The former takes $D$ to a UN set and the latter takes $D$ to a $\mathbf{U M}$ set. Hence $D$ is, by Lemma 7.1, UN $\cap \mathbf{U M}$.

The following three theorems are now, in view of the above lemmas, trivial consequences of Theorems 5.4, 5.11 and 6.8.

Theorem 7.5. Each analytic $\sigma$-monotone space $X$ contains

- $a$ UN set $E \subseteq X$ such that $\operatorname{dim}_{\mathrm{H}} E=\operatorname{dim}_{\mathrm{H}} X$,

- $a \mathbf{U N} \cap \mathbf{U M}$ set $E \subseteq X$ such that $\overline{\operatorname{dim}}_{\mathrm{H}} E=\operatorname{dim}_{\mathrm{H}} X$.

Theorem 7.6. Each analytic set $X \subseteq \mathbb{R}^{n}$ contains

- $a \mathbf{U N}$ set $E \subseteq X$ such that $\operatorname{dim}_{\mathrm{H}} E=\operatorname{dim}_{\mathrm{H}} X$,

- $a \mathbf{U N} \cap \mathbf{U M}$ set $E \subseteq X$ such that $\overline{\operatorname{dim}}_{\mathrm{H}} E=\operatorname{dim}_{\mathrm{H}} X$.

Theorem 7.7. Each metric space $X$ contains

- $a$ UN set $E \subseteq X$ such that $\operatorname{dim}_{\mathrm{H}} E \geqslant \operatorname{dim} X$,

- $a \mathbf{U N} \cap \mathbf{U M}$ set $E \subseteq X$ such that $\overline{\operatorname{dim}}_{\mathrm{H}} E \geqslant \operatorname{dim} X$.

Notice that, in contrast to Corollary 6.6, in general there is no hope that $E$ can have positive $(\operatorname{dim} X)$-dimensional Hausdorff measure. For instance, if $E \subseteq \mathbb{R}^{n}$, then $\mathcal{H}^{n}(E)$ is $\sigma$-finite, so if $\mathcal{H}^{n}(E)>0$, then the restriction of $\mathcal{H}^{n}$ to $E$ is a measure witnessing that $E$ is not $\mathbf{U N}$.

Remark 7.8. If the metric space $X$ in the above theorem is not separable, then the existence of the set $E$ is trivial: It is enough to take for $E$ a discrete set of cardinality $\omega_{1}$. One can ask if it is possible to require the set $E$ to be separable. Since there are metric spaces with positive topological dimension that have no separable subspaces with positive dimension, the answer is not obvious. Yet if $X$ is completely metrizable or, more generally, a Souslin subset of its completion, then there is a separable universally null set $E \subseteq X$ with $\operatorname{dim}_{H} E \geqslant \operatorname{dim} X$. The proof technique is quite different from the one used here and it will appear elsewhere.

\section{REMARKS}

In this section we discuss various consequences of Martin's axiom and other extra set-theoretic assumptions. The reader is supposed to be familiar with basic set theory. 
Improving $\overline{\operatorname{dim}}_{\mathrm{H}} E \geqslant \operatorname{dim}_{\mathrm{H}} X$ for UM. The existence of UN and UM sets of large dimension stems from Grzegorek's theorems that guarantee, in $\mathrm{ZFC}$, a set of large cardinality (non $\mathcal{N}$ or non $\mathcal{M}$ ) in $2^{\omega}$ that is topologically small (UN or UM). With some extra assumptions about the two cardinals one can get more, for instance, non $\mathcal{N} \leqslant$ non $\mathcal{M}$ (implied by Martin's axiom or by non $\mathcal{N}=\boldsymbol{\aleph}_{1}$ ) enables us to strengthen all of the inequalities $\overline{\operatorname{dim}}_{H} E \geqslant$ $\operatorname{dim}_{\mathrm{H}} X$ of the previous section to $\operatorname{dim}_{\mathrm{H}} E=\operatorname{dim}_{\mathrm{H}} X$ :

Proposition 8.1. Assume non $\mathcal{N} \leqslant \operatorname{non} \mathcal{M}$.

(i) Each analytic $\sigma$-monotone space $X$ contains a $\mathbf{U N} \cap \mathbf{U M}$ set $E$ such that $\operatorname{dim}_{\mathrm{H}} E=\operatorname{dim}_{\mathrm{H}} X$.

(ii) Each analytic set $X \subseteq \mathbb{R}^{n}$ contains a UN $\cap \mathbf{U M}$ set $E$ such that $\operatorname{dim}_{\mathrm{H}} E=\operatorname{dim}_{\mathrm{H}} X$.

(iii) Each metric space $X$ contains a $\mathbf{U N} \cap \mathbf{U M}$ set $E$ such that $\operatorname{dim}_{\mathrm{H}} E \geqslant$ $\operatorname{dim} X$.

Sets with no UN or UM subsets of positive dimension. It would be nice to have an example of a set with positive Hausdorff dimension such that all its UN subsets have Hausdorff dimension zero; and likewise for upper Hausdorff dimension and UM. Subject to Martin's axiom such examples are easy to construct.

Proposition 8.2. Assume Martin's axiom. Let $X$ be an analytic metric space.

(i) There is a set $S \subseteq X$ such that $\operatorname{dim}_{\mathrm{H}} S=\operatorname{dim}_{\mathrm{H}} X$, but $\overline{\operatorname{dim}}_{\mathrm{H}} E=0$ for each UN set $E \subseteq S$.

(ii) There is a set $L \subseteq X$ such that $\overline{\operatorname{dim}}_{\mathrm{H}} L=\overline{\operatorname{dim}}_{\mathrm{H}} X$, but $\overline{\operatorname{dim}}_{\mathrm{H}} E=0$ for each $\mathbf{U M}$ set $E \subseteq L$.

Proof. (i) Mutatis mutandis we may assume that $X$ is compact and $0<$ $\mathcal{H}^{s}(X)<\infty$, where $s=\operatorname{dim}_{\mathrm{H}} X$, so that $\mathcal{H}^{s}$ is a nontrivial finite diffused Borel measure on $X$.

It is well-known that Martin's axiom yields a c-Sierpiński set: a set $S \subseteq X$ such that $|S|=\mathfrak{c}$ and if $E \subseteq S$ and $\mathcal{H}^{s}(E)=0$, then $|E|<\mathfrak{c}$. In particular $\mathcal{H}^{s}(S)>0$ and thus $\operatorname{dim}_{\mathrm{H}} S=s$. On the other hand, if $E \subseteq S$ is $\mathbf{U N}$, then $\mathcal{H}^{s}(E)=0$ and thus $|E|<\mathfrak{c}$. As proved in [39], under Martin's axiom, $|E|<\mathfrak{c}$ implies $\overline{\operatorname{dim}}_{\mathrm{H}} E=0$.

(ii) It is enough to find, for each $s<\overline{\operatorname{dim}}_{\mathrm{H}} X$, a set $L \subseteq X$ such that $\overline{\operatorname{dim}}_{\mathrm{H}} L \geqslant t$ and $\overline{\operatorname{dim}}_{\mathrm{H}} E=0$ for each UM set $E \subseteq L$. Put $G=\bigcup\{U \subseteq$ $X: U$ open, $\left.\overline{\operatorname{dim}}_{H} U \leqslant s\right\}$. Then $\overline{\operatorname{dim}}_{H} G \leqslant s$ and thus each open nonempty subset $U$ of $X \backslash G$ satisfies $\overline{\operatorname{dim}}_{H} U \geqslant s$.

Martin's axiom yields a $\mathfrak{c}$-Luzin set: a set $L \subseteq X \backslash G$ such that $|L|=\mathfrak{c}$ and if $E \subseteq L$ is meager, then $|E|<\mathfrak{c}$. In particular $L$ is not meager and 
consequently, by Lemma 2.4, $\overline{\operatorname{dim}}_{\mathrm{H}} L=1$. If $E \subseteq L$ is $\mathbf{U M}$, then it is meager and thus $|E|<\mathfrak{c}$, whence $\overline{\operatorname{dim}}_{H} E=0$ by [39] again.

Other small sets. With extra set-theoretic assumptions one can make some other small sets have positive (upper) Hausdorff dimension. We consider the following classes of separable metric spaces:

- $X$ is universally small if there are no nontrivial Borel-based $c c c \sigma$-ideals on $X$.

- $X$ is a $\lambda$-set if every countable subset of $X$ is $G_{\delta}$.

- $X \subseteq Y$ is a $\lambda^{\prime}$-set in $Y$ if $X \cup D$ is a $\lambda$-set for each countable set $D \subseteq Y$.

- $X$ is a $\sigma$-set if each $F_{\sigma}$-set in $X$ is $G_{\delta}$ in $X$.

- $X$ is a $Q$-set if each subset of $X$ is $G_{\delta}$ in $X$.

The figure shows various inclusions. See [27, 28] for general reference and [38] for universally small sets.

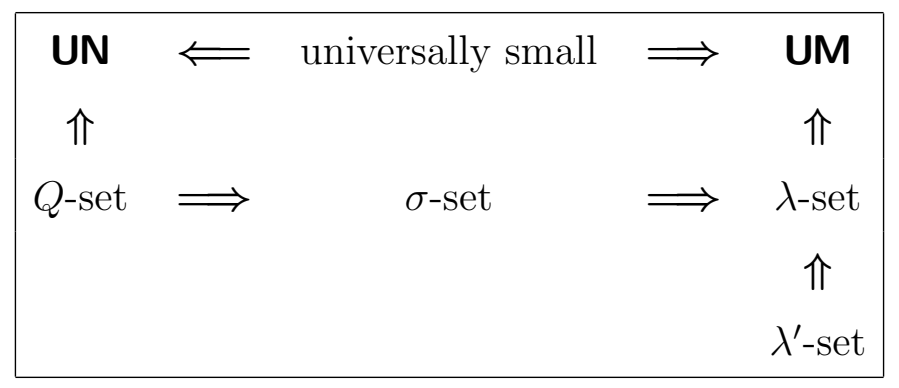

All of these classes except $\sigma$-sets are backwards closed ([27]). All of them may consistently have large Hausdorff dimension.

Proposition 8.3. It is relatively consistent with $\mathrm{ZFC}$ that each metric space $X$ contains a set $E$ with $\operatorname{dim}_{\mathrm{H}} E \geqslant \operatorname{dim} X$ and $E$ is simultaneously $Q, \lambda^{\prime}$ and universally small.

Proof. Judah and Shelah [20] have a model of "ZFC + there exists an uncountable $Q$-set + there exists a subset of reals of cardinality $\aleph_{1}$ which is not Lebesgue measurable". In other words, denoting $\mathbf{Q}$ the class of $Q$-sets, non $\mathcal{N}=\boldsymbol{\aleph}_{1} \prec \mathbf{Q}$ in the model. Let $Q$ be an uncountable $Q$-set. Let $S$ be an uncountable universally small set (there is one, see [38]). Let $L$ be an uncountable $\lambda^{\prime}$-set (there is one, see [27]). All of the three sets may be assumed to be of cardinality $\aleph_{1}$. Let $\mathcal{S}$ be the class of sets that are simultaneously $Q, \lambda^{\prime}$ and universally small. Construct a diagonal set $E \subseteq Q \times S \times L$. Since all of the three classes are backwards closed, so is $\mathcal{S}$ and $E \in \mathcal{S}$. Hence $E$ witnesses non $\mathcal{N} \prec \mathcal{S}$. Now apply Theorem 6.8 .

There are numerous variations. For instance: If non $\mathcal{N}=\aleph_{1}$, then for each analytic space there is a universally small $\lambda^{\prime}$-set $E \subseteq X$ such that 
$\operatorname{dim}_{\mathrm{H}} E=\operatorname{dim}_{\mathrm{H}} X$; if non $\mathcal{N} \leqslant \mathfrak{b}$, then there is, for each $n$, a $\lambda$-set $E \subseteq \mathbb{R}^{n}$ such that $\operatorname{dim}_{H} E=n$. There are also counterparts for $\overline{\operatorname{dim}}_{H}$ and non $\mathcal{E}$.

Most of the classes under consideration may also consistently have Hausdorff dimension zero:

Proposition 8.4. Each of the following is relatively consistent with ZFC:

(i) $\overline{\operatorname{dim}}_{\mathrm{H}} X=0$ for each $\sigma$-set,

(ii) $\overline{\operatorname{dim}}_{\mathrm{H}} X=0$ for each $\lambda^{\prime}$-set $X \subseteq \mathbb{R}$,

(iii) $\operatorname{dim}_{\mathrm{H}} X=0$ for each $\lambda$-set.

Proof. Since $\sigma$-sets are consistently countable ([26, Theorem 22]), (i) is obvious.

(ii): By [29, Theorem 1.1], it is consistent that every $\lambda^{\prime}$-set is a $\gamma$-set and in [39] it is proved that $\overline{\operatorname{dim}}_{\mathrm{H}} X=0$ for every $\gamma$-set $X$.

(iii): By [28, Theorem 22], if $\omega_{2}$ Cohen reals are added to a model of $\mathrm{CH}$, then each $\lambda$-set in the extension is of cardinality $\boldsymbol{\aleph}_{1}$ or less. Also $\operatorname{cov} \mathcal{M}=\aleph_{2}=\mathfrak{c}$ in the model. In particular, $|X|<\operatorname{cov} \mathcal{M}$ for each $\lambda$-set. But if $X$ is a separable metric space and $|X|<\operatorname{cov} \mathcal{M}$, then $\mathcal{H}^{s}(X)=0$ for each $s>0$ (see e.g. $[14,534 \mathrm{~B}(\mathrm{c})]$ ), and therefore $\operatorname{dim}_{H} X=0$.

Proposition 8.5. There is a $\lambda$-set $X$ such that $\overline{\operatorname{dim}}_{\mathrm{H}} X=\infty$.

Proof. Provide the set of irrationals $\omega^{\omega}$ with the (variation of) Baire metric $\rho(f, g)=2^{-n(f, g)}$ (cf. the first paragraph of Section 3$)$. Let $X \subseteq \omega^{\omega}$ be an unbounded set of cardinality $\mathfrak{b}$. Rothberger [32] (or see [28, Theorem 21]) shows that $X$ is a $\lambda$-set. If $\overline{\operatorname{dim}}_{\mathrm{H}} X<\infty$, then it is contained in a $\sigma$-compact set. But that is not possible, since $X$ is unbounded.

More small sets. Some notions of small sets are defined via addition of reals. For sets $X, A \subseteq 2^{\omega}$ write $X+A=\{x+a: x \in X, a \in A\}$; the addition is componentwise modulo 2 .

- $X$ is strongly null if for each sequence $\varepsilon_{n} \searrow 0$ there is a cover $\left\{U_{n}\right\}$ of $X$ such that $\operatorname{diam} U_{n} \leqslant \varepsilon_{n}$ for all $n$. It is well-known that $X \subseteq 2^{\omega}$ is strongly null iff $X+M \neq 2^{\omega}$ for each $M \in \mathcal{M}$ (Galvin-MycielskiSolovay Theorem, see [19] or [27])

- $X \subseteq 2^{\omega}$ is strongly meager if $X+N \neq 2^{\omega}$ for each $N \in \mathcal{N}$.

- $X \subseteq 2^{\omega}$ is $\mathcal{M}$-additive if $X+M \in \mathcal{M}$ for each $M \in \mathcal{M}$.

- $X \subseteq 2^{\omega}$ is strongly meager if $X+N \in \mathcal{N}$ for each $N \in \mathcal{N}$.

The figure shows the inclusions. For a general reference, see [1]. The horizontal inclusions are trivial. As to the vertical ones, strongly null $\Rightarrow \mathbf{U N}$ is folklore and fairly easy, $\mathcal{N}$-additive $\Rightarrow \mathcal{M}$-additive is due to Shelah [35] and $\mathcal{M}$-additive $\Rightarrow \mathbf{U M}$ is proved in [39]. 


\begin{tabular}{|ccc|}
\hline UM & UN \\
$\Uparrow$ & $\Uparrow$ \\
$\mathcal{M}$-additive & $\Longrightarrow$ & strongly null \\
$\Uparrow$ & & \\
$\mathcal{N}$-additive & $\Longrightarrow$ & strongly meager \\
\hline
\end{tabular}

In the sequel, $2^{\omega}$ is equipped with the metric of the Cantor cube $\mathbb{C}\left(\frac{1}{2}, \frac{1}{2}\right)$.

Proposition 8.6. (i) If $X$ is strongly null, then $\operatorname{dim}_{\mathrm{H}} X=0$.

(ii) If $X \subseteq 2^{\omega}$ is $\mathcal{N}$-additive or $\mathcal{M}$-additive, then $\overline{\operatorname{dim}}_{\mathrm{H}} X=0$.

Proof. (i) is due to Besicovitch [3]. (ii) is proved in [39].

Proposition 8.7. Each of the following is relatively consistent with ZFC:

(i) $\overline{\operatorname{dim}}_{\mathrm{H}} X=0$ for each $X \subseteq 2^{\omega}$ strongly meager.

(ii) $\overline{\operatorname{dim}}_{\mathrm{H}} X=0$ for each $X$ strongly null.

Proof. By [5] it is consistent that each strongly meager set is countable. By [22] it is consistent that each strongly null set is countable.

Proposition 8.8. Assume Martin's axiom.

(i) There is a strongly null set $X \subseteq 2^{\omega}$ such that $\overline{\operatorname{dim}}_{\mathrm{H}} X=1$,

(ii) there is a strongly meager set $X \subseteq 2^{\omega}$ such that $\operatorname{dim}_{\mathrm{H}} X=1$.

Proof. (i) Take for $X$ the c-Luzin set of Proposition 8.2. By [18], (or see [1, Lemma 8.2.4]), each $\mathfrak{c}$-Luzin set is strongly null.

(ii) Take for $X$ the $\mathfrak{c}$-Sierpiński set of Proposition 8.2. Pawlikowski showed that a Sierpiński set is strongly meager. The proof [1, Theorem 8.5.13] of Pawlikowski's theorem can be easily adapted to show that under Martin's axiom a $\mathfrak{c}$-Sierpiński set is strongly meager.

\section{REFERENCES}

1. Tomek Bartoszyński and Haim Judah, Set theory, A K Peters Ltd., Wellesley, MA, 1995, On the structure of the real line. MR 1350295 (96k:03002)

2. Tomek Bartoszyński and Saharon Shelah, Closed measure zero sets, Ann. Pure Appl. Logic 58 (1992), no. 2, 93-110. MR 1186905 (94b:03084)

3. A. S. Besicovitch, Concentrated and rarified sets of points, Acta Math. 62 (1933), no. 1, 289-300. MR 1555386

4. __ On existence of subsets of finite measure of sets of infinite measure, Nederl. Akad. Wetensch. Proc. Ser. A. 55 = Indagationes Math. 14 (1952), 339-344. MR $0048540(14,28 \mathrm{e})$

5. Timothy J. Carlson, Strong measure zero and strongly meager sets, Proc. Amer. Math. Soc. 118 (1993), no. 2, 577-586. MR 1139474 (94b:03086) 
6. R. O. Davies, Subsets of finite measure in analytic sets, Nederl. Akad. Wetensch. Proc. Ser. A. 55 = Indagationes Math. 14 (1952), 488-489. MR 0053184 (14,733g)

7. R. Engelking, Dimension theory, North Holland, Amsterdam, 1978.

8. __ General topology, Heldermann Verlag, Berlin, 1989.

9. K. J. Falconer and G. R. Grimmett, On the geometry of random Cantor sets and fractal percolation, J. Theoret. Probab. 5 (1992), no. 3, 465-485. MR 1176432 (94b:60115)

10. Kenneth Falconer, Techniques in fractal geometry, John Wiley \& Sons Ltd., Chichester, 1997. MR 1449135 (99f:28013)

11. Kenneth J. Falconer, The geometry of fractal sets, Cambridge Tracts in Mathematics, vol. 85, Cambridge University Press, Cambridge, 1986. MR 867284 (88d:28001)

12. , Fractal geometry, second ed., John Wiley \& Sons Inc., Hoboken, NJ, 2003, Mathematical foundations and applications. MR 2118797 (2006b:28001)

13. D. H. Fremlin, Measure theory, vol. 4, Torres Fremlin, 2003.

14. Measure theory, vol. 5, Torres Fremlin, 2008?

15. E. Grzegorek, Solution to a problem of Banach on $\sigma$-fields without continuous measures, Acad. Pol. Sci. (1980), no. 28, 7-10.

16. J. D. Howroyd, On Hausdorff and packing dimension of product spaces, Math. Proc. Cambridge Philos. Soc. 119 (1996), no. 4, 715-727. MR 1362951 (96j:28006)

17. W. Hurewicz and H. Wallman, Dimension theory, Princeton, 1941.

18. Jaime I. Ihoda, Strong measure zero sets and rapid filters, J. Symbolic Logic 53 (1988), no. 2, 393-402. MR 947847 (89h:03083)

19. Haim Judah (ed.), Set theory of the reals, Israel Mathematical Conference Proceedings, vol. 6, Ramat Gan, Bar-Ilan University Gelbart Research Institute for Mathematical Sciences, 1993. MR 1234275 (94b:03010)

20. Haim Judah and Saharon Shelah, Q-sets, Sierpiński sets, and rapid filters, Proc. Amer. Math. Soc. 111 (1991), no. 3, 821-832. MR 1045594 (91f:03105)

21. Alexander S. Kechris, Classical descriptive set theory, Graduate Texts in Mathematics, vol. 156, Springer-Verlag, New York, 1995. MR 1321597 (96e:03057)

22. Richard Laver, On the consistency of Borel's conjecture, Acta Math. 137 (1976), no. 3-4, 151-169. MR 0422027 (54 \#10019)

23. Jiří Matoušek, On Lipschitz mappings onto a square, The mathematics of Paul Erdös, II, Algorithms Combin., vol. 14, Springer, Berlin, 1997, pp. 303-309. MR 1425223 (98g:28009)

24. Pertti Mattila, Geometry of sets and measures in Euclidean spaces, Cambridge Studies in Advanced Mathematics, vol. 44, Cambridge University Press, Cambridge, 1995, Fractals and rectifiability. MR 1333890 (96h:28006)

25. Pertti Mattila and R. Daniel Mauldin, Measure and dimension functions: measurability and densities, Math. Proc. Cambridge Philos. Soc. 121 (1997), no. 1, 81-100. MR 1418362 (97k:28014)

26. Arnold W. Miller, On the length of Borel hierarchies, Ann. Math. Logic 16 (1979), no. 3, 233-267. MR 548475 (80m:04003)

27. _ Special subsets of the real line, Handbook of set-theoretic topology, NorthHolland, Amsterdam, 1984, pp. 201-233. MR 776624 (86i:54037)

28. Special sets of reals, Set theory of the reals (Ramat Gan, 1991), Israel Math. Conf. Proc., vol. 6, Bar-Ilan Univ., Ramat Gan, 1993, pp. 415-431. MR 1234286 (95a:03064)

29. _ On $\lambda^{\prime}$-sets, Topology Proc. 28 (2004), no. 1, 179-187, Spring Topology and Dynamical Systems Conference. MR 2105456 (2005h:03093) 
30. Aleš Nekvinda and Ondřej Zindulka, Monotone spaces, (2008), preprint.

31. C. A. Rogers, Hausdorff measures, Cambridge University Press, Cambridge, 1970.

32. F. Rothberger, Sur un ensemble toujours de première categorie qui est dépourvu de la propriété $\lambda$, Fund. Math. 32 (1939), 294-300.

33. A. Schief, Separation properties for self-similar sets, Proc. Amer. Math. Soc. 122 (1994), 111-115.

34. , Self-similar sets in complete metric spaces, Proc. Amer. Math. Soc. 124 (1996), no. 2, 481-490.

35. Saharon Shelah, Every null-additive set is meager-additive, Israel J. Math. 89 (1995), no. 1-3, 357-376. MR 1324470 (96j:04002)

36. Piotr Zakrzewski, Universally meager sets II, (200?), to appear.

37. _ Universally meager sets, Proc. Amer. Math. Soc. 129 (2001), no. 6, 17931798 (electronic). MR 1814112 (2001m:03097)

38. - On a construction of universally small sets, Real Anal. Exchange 28 (2002/03), no. 1, 221-226. MR 1973982 (2004c:03061)

39. Ondřej Zindulka, Small sets vs. fractal dimensions, to appear.

40. _ Dimension zero vs. measure zero, Proc. Amer. Math. Soc. 128 (2000), no. 6 , 1769-1778.

41. __ Small opaque sets, Real Analysis Exchange 28 (2003), no. 2, 1-15.

Department of Mathematics, Faculty of Civil Engineering, Czech Technical University, Thákurova 7, 16000 Prague 6, Czech Republic

E-mail address: zindulka@mat.fsv.cvut.cz

URL: http://mat.fsv.cvut.cz/zindulka 